\title{
Making Hydrogels Stronger through Hydrophilicity-Hydrophobicity Transformation, Thermoresponsive Morphomechanics and Crack Multifurcation
}

YUBING HU, Lucile Barbier, Zhao Li, Xiaofan Ji, Heiva Le Blay, Junkai Liu, Jacky W. Y. Lam, Alba Marcellan, Ben Zhong Tang

Submitted date: 11/05/2020 - Posted date: 12/05/2020

Licence: CC BY-NC-ND 4.0

Citation information: HU, YUBING; Barbier, Lucile; Li, Zhao; Ji, Xiaofan; Blay, Heiva Le; Liu, Junkai; et al. (2020): Making Hydrogels Stronger through Hydrophilicity-Hydrophobicity Transformation, Thermoresponsive Morphomechanics and Crack Multifurcation. ChemRxiv. Preprint.

https://doi.org/10.26434/chemrxiv.12279686.v1

The development of mechanically strong, flexible and crack-resistant hydrogels is of great academic and practical significance and demands for the biomimetic exploration of energy dissipation pathways. The rational design of strong hydrogels is also limited by insufficient mechanism study, resulting from the lack of powerful technique to "see" hydrogels at morphological level. Herein, we constructed a thermoresponsive mechanically strong hydrogel from poly(N-isopropylacrylamide) (PNIPAM) and poly(N,N-dimethylacrylamide). Its hydrophilicity-hydrophobicity transformation and composition-dependent microphase separation are directly visualized by using luminogens with aggregation-induced emission as fluorescent indicators. Based on the morphological observation and mechanical measurements, the concept of morphomechanics with a comprehensive mechanism clarification is proposed. In this regard, thermoresponsive strengthened mechanical properties are attributed to the entanglement of PNIPAM chains and the formation of multiple noncovalent interactions, mainly hydrogen bonds. The enhanced fracture energy by crack multifurcation is related to the disruption of weak interfaces between two separated phases.

File list (2)

Manuscript for chemrxiv.pdf (1.57 MiB)

view on ChemRxiv • download file 


\title{
Making Hydrogels Stronger through Hydrophilicity-Hydrophobicity Transformation, Thermoresponsive Morphomechanics and Crack Multifurcation
}

\author{
Yubing $\mathrm{Hu}^{1,2}$, Lucile Barbier ${ }^{3}$, Zhao Li ${ }^{1,2}$, Xiaofan $\mathrm{Ji}^{1,2}$, Heiva Le Blay ${ }^{3}$, Junkai Liu ${ }^{1,2}$, Jacky W. Y. Lam ${ }^{1,2, *}$, \\ Alba Marcellan ${ }^{3, *}$, and Ben Zhong Tang ${ }^{1,2,4, *}$
}

\begin{abstract}
The development of mechanically strong, flexible and crack-resistant hydrogels is of great academic and practical significance and demands for the biomimetic exploration of energy dissipation pathways. The rational design of strong hydrogels is also limited by insufficient mechanism study, resulting from the lack of powerful technique to "see" hydrogels at morphological level. Herein, we constructed a thermoresponsive mechanically strong hydrogel from poly( $N$-isopropylacrylamide) (PNIPAM) and poly( $N, N$-dimethylacrylamide). Its hydrophilicityhydrophobicity transformation and composition-dependent microphase separation are directly visualized by using luminogens with aggregation-induced emission as fluorescent indicators. Based on the morphological observation and mechanical measurements, the concept of morphomechanics with a comprehensive mechanism clarification is proposed. In this regard, thermoresponsive strengthened mechanical properties are attributed to the entanglement of PNIPAM chains and the formation of multiple noncovalent interactions, mainly hydrogen bonds. The enhanced fracture energy by crack multifurcation is related to the disruption of weak interfaces between two separated phases.
\end{abstract}

Keywords: hydrophilicity-hydrophobicity transformation, microphase separation, crack multifurcation, aggregation-induced emission

${ }^{1}$ Department of Chemistry, The Hong Kong University of Science and Technology, Clear Water Bay, Kowloon, Hong Kong, China

${ }^{2}$ Hong Kong Branch of Chinese National Engineering Research Center for Tissue Restoration and Reconstruction, Institute for Advanced Study and HKUST-Shenzhen Research Institute, The Hong Kong University of Science and Technology, Clear Water Bay, Kowloon, Hong Kong, China

${ }^{3}$ ESPCI ParisTech, PSL Research University, Sorbonne Universités, UPMC Univ. Paris 06, Laboratoire Sciences et Ingénierie de la Matière Molle CNRS UMR CNRS 7615, 10 Rue Vauquelin F-75231, Paris 5, France

${ }^{4}$ Center for Aggregation-Induced Emission, SCUT-HKUST Joint Research Institute, State Key Laboratory of Luminescent Materials and Devices, South China University of Technology, Guangzhou 510640, China 
Hydrogels have been widely applied in tissue engineering ${ }^{[1]}$ drug delivery ${ }^{[2-3]}$, and biomimetic models ${ }^{[4-5]}$, due to their similar wet and soft nature with living organism. Recent progress has inspired various advanced technologies in biomedical devices ${ }^{[6-7]}$, soft robotics ${ }^{[8-9]}$, optics ${ }^{[10-11]}$, stretchable ionotronics $^{[12]}$, and bioelectronics ${ }^{[13-14]}$, and yet their practical applications are still limited. In contrast, organism tissues have been optimized with diverse structure, tunable topography and adaptable mechanical properties to realize their functionalities in complex environment after million years of evolution. ${ }^{[15-16]}$ Many forced-related tissues (spider silk, muscle and tendon) are assembled by multiple ordered fibers and mainly constructed by the intra/interchain noncovalent interactions of hydrophilic proteins. ${ }^{[17]}$ These swelling-stable tissues exhibit high-strength and flexible mechanical properties, which are mainly owing to the structural variation of proteins including unfolding of $\beta$-sheet assembly and uncoiling of tropocollagen ${ }^{[18]}$. These tissues have a natural tendency to tear along the interfaces between fibers to prevent injury to the entire tissue under excessive tension (Figure 1). ${ }^{[19-21]}$ These phenomena are intriguing to synthetic scientists. However, it is a big challenge to fully understand the relationship between their complex structure and mechanical properties, and further utilize them in hydrogels.

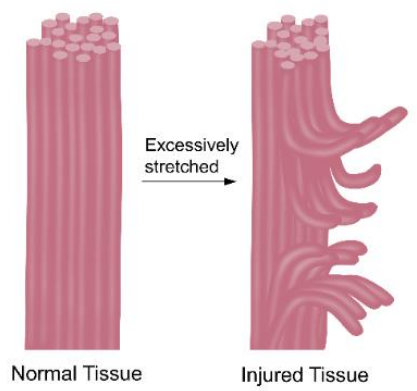

Figure 1. Simplified tearing model of fiber-assembled tissues under excessive tension.

To overcome the mechanical limitation and meet the everincreasing requirements, continuous effort has been devoted to the development of high-strength hydrogel. ${ }^{[22-23]}$ Emerging strategies has been employed to construct high-strength hydrogels, such as double network hydrogels ${ }^{[24]}$, sliding-ring hydrogels ${ }^{[25]}$, nanocomposite hydrogels ${ }^{[26]}$ topological hydrogels ${ }^{[27]}$, ionically crosslinked hydrogels ${ }^{[28]}$, and so on. Great achievement has been reached, while the exploration of smart hydrogel should be further promoted through learning from natural structure, topography, and versatile functionalities. ${ }^{[29]}$ The rational design of next-generation hydrogels is also affected by the insufficient study of strengthened mechanism. The current accepted mechanism models of strengthened hydrogels are always deduced from microscopic network structure and macroscopic properties. ${ }^{[30-}$ ${ }^{33]}$ In other words, the effect of morphological conformation and assembly on mechanical strength remains at the hypothesis level. ${ }^{[34]}$ Hence, it is essential to develop an effective technique to "see" the mesoscale morphologies at hydrated state and clarify their roles in energy dissipation.

Biomimetic approach offers a broad platform to explore pathways to dissipate energy for high-strength hydrogels. ${ }^{[35]}$ From unfolding of $\beta$-sheet assembly to tearing between fibers, morphomechanics can be proposed to describe such phenomena of sacrificing the noncovalent interactions or weak interfaces to preserve the integrity of the whole. This is extended from the concept of mechanochemistry ${ }^{[36]}$ and enjoys various advantages of simple fabrication, structure diversity and reversibility manipulation. Exploring strategies to strengthen hydrogel is necessary and moreover, it is attractive to figure out the mechanism by visualizing the strengthening process. Fluorescence technique offers direct visualization approach in a simple, sensitive, high-contrast and onsite manner. However, many traditional fluorophores exhibit weak or no emission in the solid state and aqueous environment due to aggregation-caused quenching. ${ }^{[37]}$ Fortunately, this negative effect can be overcome by luminogens with aggregation-induced emission (AIEgens), which are generally non-emissive in dilute solutions but emit intensely in the aggregated state due to the restriction of intramolecular motions. ${ }^{[38]}$ AIEgens are often embedded with electron donor (D) and acceptor (A) units to provide polarityresponsive emission change, thanks to twisted intramolecular charge transfer (TICT) effect. ${ }^{[39]}$

Design and characterization of TICT-active AIEgen. To visualize the morphological change of stimuli-responsive hydrogels, we designed and acquired a new AIEgen named TVPA with chemical structure as shown in Figure 2a. The electron-donating triphenylamine moiety and the electronwithdrawing tetrasubstituted $N$-(acryloyloxy)ethyl pyridinium unit are connected to ethylene core, which is to endow TVPA with molecular rotors, D-A structure and ionic character. The three-step synthetic route and detailed procedures are provided in Figure S1. The reaction intermediates and TVPA were characterized by NMR and high-resolution mass spectroscopies with satisfactory results (Figures S3-10). To further understand the molecular characteristics, the theoretical frontier molecular orbital distribution was calculated by density functional theory calculations [DFT, m062x/6-311G (d, p)]. As shown in Figure $2 \mathrm{a}$, the highest occupied molecular orbital (HOMO) mainly localizes in the triphenylamine unit and the lowest unoccupied molecular orbital (LUMO) mainly locates in the pyridinium unit. Such electron separation of HOMO and LUMO indicates a $\pi-\pi^{*}$ transition with a degree of charge transfer character.

To verify the desired optical properties of TVPA, we first investigated its photoluminescent (PL) spectra and quantum yields (QYs) in water, glycerol and the solid state. As shown in Figure 2b, water-soluble TVPA exhibits weak red emission in dilute aqueous solution but emits intensely in viscous glycerol and the solid state with emission enhanced by 87 and 54 times, respectively. The corresponding QYs of TVPA are also increased from $0.4 \%$ to $10.7 \%$ and $6.1 \%$, confirming the AIE characteristic of TVPA from the restriction of intramolecular motions. Considering the $\mathrm{D}-\mathrm{A}$ structure of TVPA, we recorded its PL spectra and fluorescent photos in different solvents and polymer films with various polarities. Figure 2c demonstrates a remarkable solvatochromic effect of TVPA with emission color gradually shifting from deep blue $\left(\lambda_{\mathrm{em}}=436 \mathrm{~nm}\right)$ to red $\left(\lambda_{\mathrm{em}}=610 \mathrm{~nm}\right)$ when changing the solvent from toluene to methanol. Polymer films with TVPA also display the evident bathochromic shift from blue emission $\left(\lambda_{\mathrm{em}}=457 \mathrm{~nm}\right)$ in non-polar polybutadiene to red emission $\left(\lambda_{\mathrm{em}}=621 \mathrm{~nm}\right)$ in polar poly(ethylene glycol) $(\mathrm{PEG})$ (Figure 2d). Such polarity-responsive behaviors clearly manifest the TICT characteristic of TVPA. In short, we 
successfully achieve TVPA with desired strong emission in the aggregate state and high sensitivity to environmental polarity with a broad response window, indicating a promising fluorescent sensor to the crowdedness and polarity change in hydrogels.
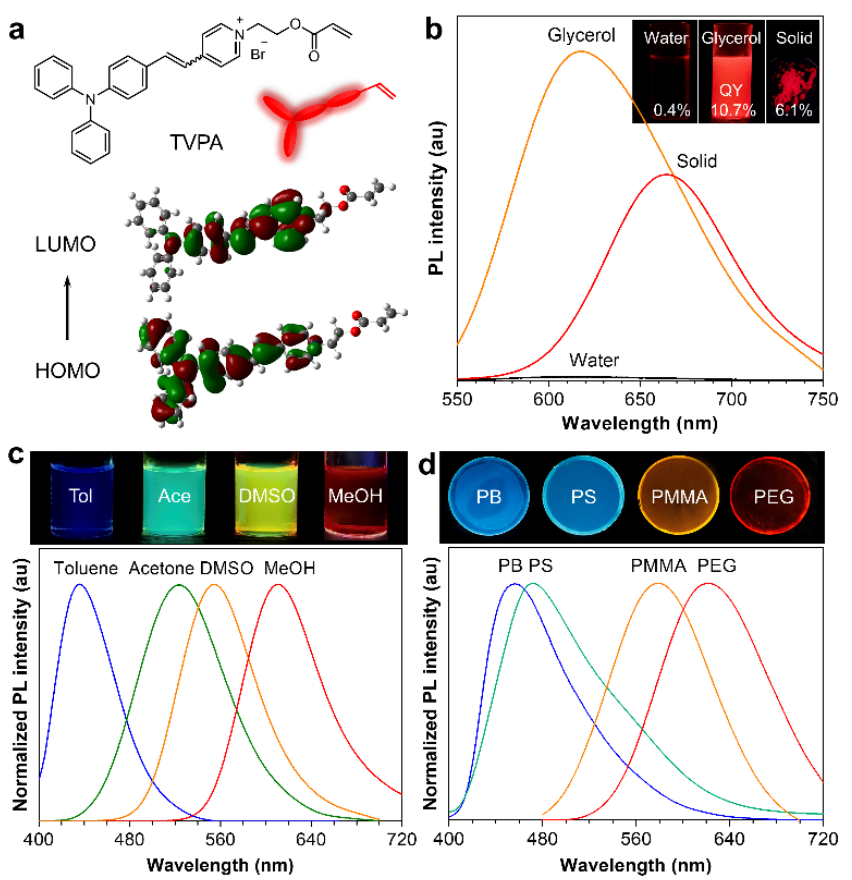

Figure 2. Optical properties of TVPA. a, Chemical structure of TVPA and its theoretical calculated molecular orbital amplitude plots of HOMO and LUMO. b, Emission spectra of TVPA in water $(10 \mu \mathrm{M})$, viscous glycerol (10 $\mu \mathrm{M})$ and the solid state. Inset: The corresponding fluorescent photographs and quantum yield (QY) of TVPA. c,d, Fluorescent photographs and emission spectra of TVPA (c) in solvents with different polarities: Toluene (Tol), acetone (Ace), dimethyl sulfoxide (DMSO) and methanol (MeOH) and (d) in spin-coated polymer films with various polarities: polybutadiene (PB), polystyrene (PS), poly(methyl methacrylate) (PMMA) and poly(ethylene glycol) (PEG).

Stimuli-responsive hydrogels with fluorescent indicators. Based on the previous efforts in phase-separated hydrogels ${ }^{[40-42]}$, we attempted to incorporate TVPA in the chemically crosslinked poly $(N$-isopropylacrylamide) (PNIPAM) network grafted with $\operatorname{poly}(N, N$ dimethylacrylamide) (PDMA) as schematically illustrated in Figure 3a. The KPS/TEMED-initiated free radical polymerization of NIPAM monomers, TVPA and PDMA macromonomers with $N, N^{\prime}$-methylenebisacrylamide (MBA) crosslinkers were designed to obtain the as-prepared hydrogels. We hypothesize that PNIPAM network can reversibly alternate between the as-prepared state and the strengthened state under thermal stimuli below and above lower critical solution temperature $\left(\mathrm{LCST}, T_{\mathrm{c}} \approx 32{ }^{\circ} \mathrm{C}\right)^{[43]}$, respectively. Well-hydrated PNIPAM chains should undergo a coil-to-globule transition and collapse into hydrophobic PNIPAM-rich domains above LCST, while PDMA chains could impede the shrinkage of the bulk hydrogels due to its high water-affinity. Meanwhile, the covalently incorporated TVPA is expected to exhibit a fluorescent response from dark red-emission in the extended hydrophilic PNIPAM chains to bright blue-emission in the crowded hydrophobic PNIPAMrich domains. Using this design principle, three hydrogels (GN2D3, GN3D3 and GN6D3) were achieved from different weight fractions of NIPAM and PDMA macromonomers (240 $\mathrm{kg} / \mathrm{mol}$ ) with same crosslink density and elastically active chains (Table S1). For comparison purposes, GN and GD hydrogels were prepared from NIPAM monomers and DMA monomers with TVPA, respectively. The macroscopic properties of three as-prepared hydrogels in different temperature were investigated by swelling degree, transmittance and PL measurements. Compared to the swelling degree of as-prepared hydrogels, the equilibrium weight of GN2D3, GN3D3 and GN6D3 increased by $1208 \%$, $760 \%, 485 \%$ at $20{ }^{\circ} \mathrm{C}$ and $68 \%, 3 \%,-58 \%$ at $60{ }^{\circ} \mathrm{C}$, respectively (Table S1). Such swelling behavior implies that they successfully inherit the thermoresponsive properties from LCST-type PNIPAM and their swelling ability is tunable by changing the weight fraction of PNIPAM and PDMA. Taking account of their absorption spectra, we then measured the transmittance change at $600 \mathrm{~nm}$ as a function of temperature from $20{ }^{\circ} \mathrm{C}$ to $60{ }^{\circ} \mathrm{C}$ (Figure S11). All the transmittance percentage of GN2D3, GN3D3 and GN6D3 drops drastically in the range of $24-34{ }^{\circ} \mathrm{C}$ with visible transparent to opaque change. Their similar transmittance behaviors indicate the conversion from homogeneous to heterogeneous distributions of PDMA and PNIPAM, resulted from the phase separation above LCST.

Since the incorporation of TVPA provides an access to use fluorescence technique in hydrogels, we carefully studied the emission properties of the three hydrogels at as-prepared state and after heating for $30 \mathrm{~min}$. As indicated in Figure $3 \mathrm{~b}$, all the PL spectra of GN2D3, GN3D3 and GN6D3 display remarkable emission intensity and color changes below and above $T_{\mathrm{c}}$. Upon the thermal stimuli from $20^{\circ} \mathrm{C}$ to $60{ }^{\circ} \mathrm{C}$, the emission maximum of the PL spectra increases by up to 17.4 times and approximately shifts from $620 \mathrm{~nm}$ to $480 \mathrm{~nm}$. The reversible fluorescence change between dark orange and bright blue can be observed by naked eyes. Among the three hydrogels, GN6D3 with the highest composition of PNIPAM is most intensified and least blue-shifted possibly contributed by its macroscopic shrinkage and the most loosely collapsed PNIPAM chains, respectively. To better understand the source of fluorescence change, we then studied the PL spectra of GN and GD at $20^{\circ} \mathrm{C}$ and $60{ }^{\circ} \mathrm{C}$ in Figure $3 \mathrm{c}$ and $3 \mathrm{~d}$. They show the similar thermally induced fluorescence change existed in GN but not in GD, and the emission color of GN above $T_{\mathrm{c}}$ is close to that in traditional non-polar polymers. Referring to the AIE and TICT characteristics of TVPA, such enhanced and blue-shifted fluorescence verifies the coil-toglobule conformational changes and implies the transformation from hydrophilic PNIPAM chains to hydrophobic PNIPAM domains above LCST. In other words, TVPA can serve as a simple and effective indicator to microenvironmental changes in hydrogels, especially to the transformation from molecular hydrophilicity to morphological hydrophobicity.

We presented more proof-of-concept experiments in stimuli-responsive gelation of TVPA-containing synthetic polymers, synthetic-natural complex, and natural polymers. In Figure 3e, Poly(ethylene glycol)-block-poly(propylene glycol)-block-poly(ethylene glycol) copolymers (PEG-PPGPEG) in aqueous solution displays thermoresponsive gelation with gradually blue-shifted emission upon heating from $5{ }^{\circ} \mathrm{C}$ to $60{ }^{\circ} \mathrm{C}$. The mixture of weakly red-emissive $\alpha$-cyclodextrin $(\alpha-\mathrm{CD})$ and PEG solutions is gelled after the complex formation and the $\alpha$-CD-PEG inclusion complexes exhibit strong orange emission (Figure 3f). The gelation of chitosan solution is induced upon the addition and diffusion of sodium 

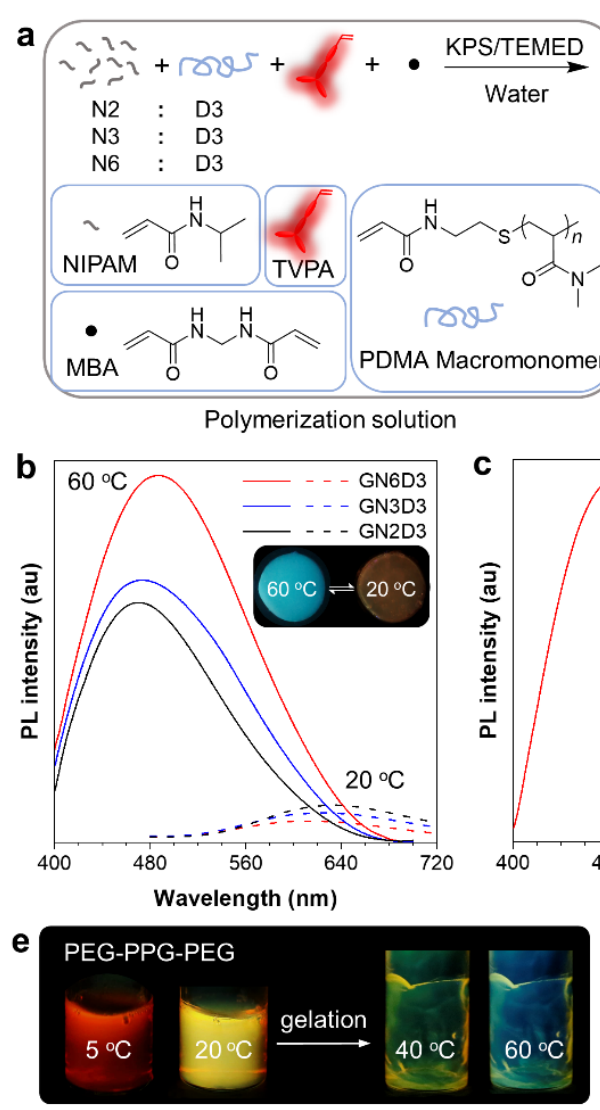
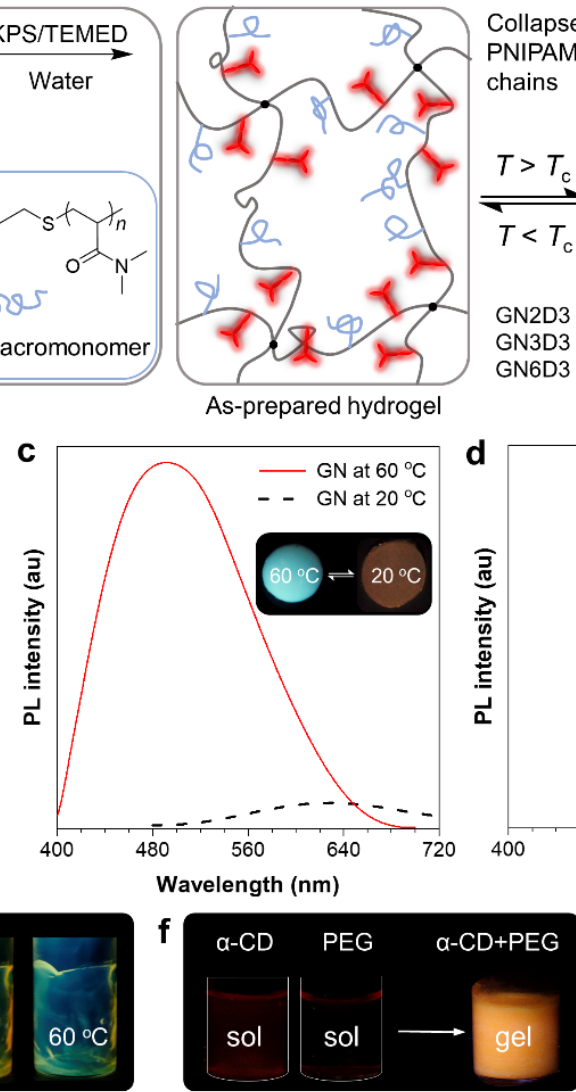

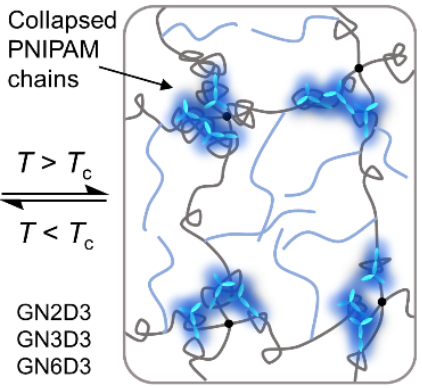

Strengthened hydrogel
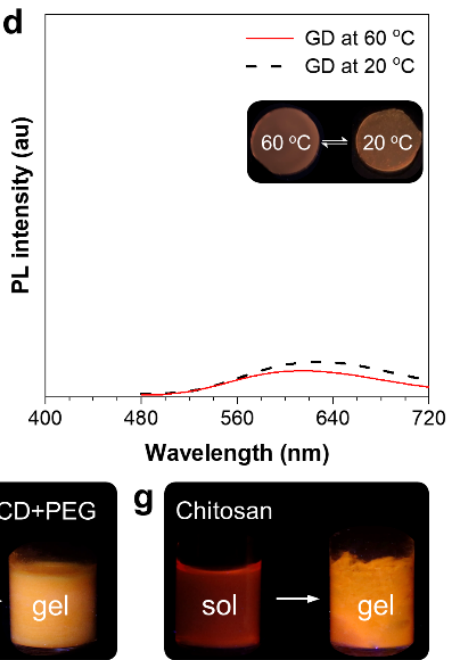

Figure 3. Schematic illustration of design principle and visualization of hydrophilicity-hydrophobicity transformation. a, TVPA was incorporated into the crosslinked NIPAM polymer network during the free radical polymerization of NIPAM, TVPA and PDMA macromonomers with MBA crosslinkers. The collapse of the resulting PNIPAM network is induced above its lower critical solution temperature (LCST, $T_{\mathrm{c}}$ ) and the emission of the incorporated TVPA is expected to be enhanced and blue-shifted in the crowded and hydrophobic PNIPAM domains. Three hydrogels (GN2D3, GN3D3, GN6D3) were prepared with different weight fractions of NIPAM and PDMA. b-d, Emission spectra of TVPA incorporated GN2D3, GN3D3, GN6D3 (b), GD (c) and GN (d) hydrogels at $20^{\circ} \mathrm{C}$ and $60{ }^{\circ} \mathrm{C}$ exciated at $380 \mathrm{~nm}$. Inset: fluorescent photos of hydrogels at $20{ }^{\circ} \mathrm{C}$ and $60{ }^{\circ} \mathrm{C}$ under $365 \mathrm{~nm} \mathrm{UV}$ light. e-f, Emission response to the transformation from molecular hydrophilicity to morphology hydrophobicity in various stimuli-responsive gelation. e, PEG-PPG-PEG solution is gelled at $40^{\circ} \mathrm{C}$ and its thermoresponsive emission color is gradually blue-shifted with temperature increasing. $\mathbf{f}$, Mixture of PEG and $\alpha-C D$ solutions is gelled after the complex formation for $24 \mathrm{~h}$. $\mathbf{g}$, Chitosan solution ( $\mathrm{pH}$ 5.6) is gelled upon the addition of sodium hydroxide solution $(0.01 \mathrm{M})$.

hydroxide solution with emission color changing from red to orange (Figure 3g). Collectively, these emission behaviors represent that TVPA could sensitively detect the universal hydrophilicity-hydrophobicity transformation in stimuliresponsive hydrogels and differentiate the degree of transformation by varying emission color.

Fluorescent imaging of microphase separated hydrogels. Based on the preliminary results, we employed the fluorescence microscope to systematically investigate the thermoreponsive and composition-dependent phase separation of PNIPAM and PDMA domains in mesoscopic scale. The fluorescent images of GN3D3 at preparation state, after immersing in water at $60{ }^{\circ} \mathrm{C}$ for $30 \mathrm{~min}$ and after overnight recovery at $20{ }^{\circ} \mathrm{C}$ are selected as a representative for thermoreponsive phase separation. As shown in Figure $4 \mathrm{a}-\mathrm{c}$, all fluorescent images display Newton's rings of different sizes with multicolor fringes. This can be attributed to the light interference caused by optical path difference due to the different refractive index of PNIPAM/PDMA and water, and the light refraction to different degrees relevant to the spectral distribution. Applying knowledge of optics to this system, we can draw two theoretical guidelines: 1 . The total numbers of interference fringes in Newton's rings is determined by the size of the corresponding object; 2 . The distance between two consecutive fringes of same color, called as fringe spacing, depends on the difference in refractive index between the PNIPAM/PDMA and water. Resulted showed that their emission colors switch between chromatic-orange and chromatic-blue under thermal stimuli, which is consistent with emission maximum and peak distribution in Figure 3b. The interference patterns in Figure 4a display the continuous irregular combinations of Newton's rings, which is associated with heterogenous distribution of partially hydrated polymer phase and aqueous phase. Very interestingly, a morphological pattern with two distinct regions is generated by heating GN3D3 above LCST when the volume change of hydrogels at macroscopic level is negligible (Figure 4b). One region displays as plenty of strongly emissive Newton's rings, where well-hydrated PNIPAM chains collapse into dehydrated PNIPAM domains, resulting in a large difference in refractive index between PNIPAM domains and water. Another region displays as uniform weakly emissive matrix, where lowly hydrated PDMA chains swell into highly hydrated PDMA domains, causing comparable refractive index of PDMA domains to water. In this way, the incorporated TVPA as a fluorescent indicator offers a simple, high-contrast and onsite access to visualize the microphase separation of PNIPAM-rich and PDMA-rich domains. 

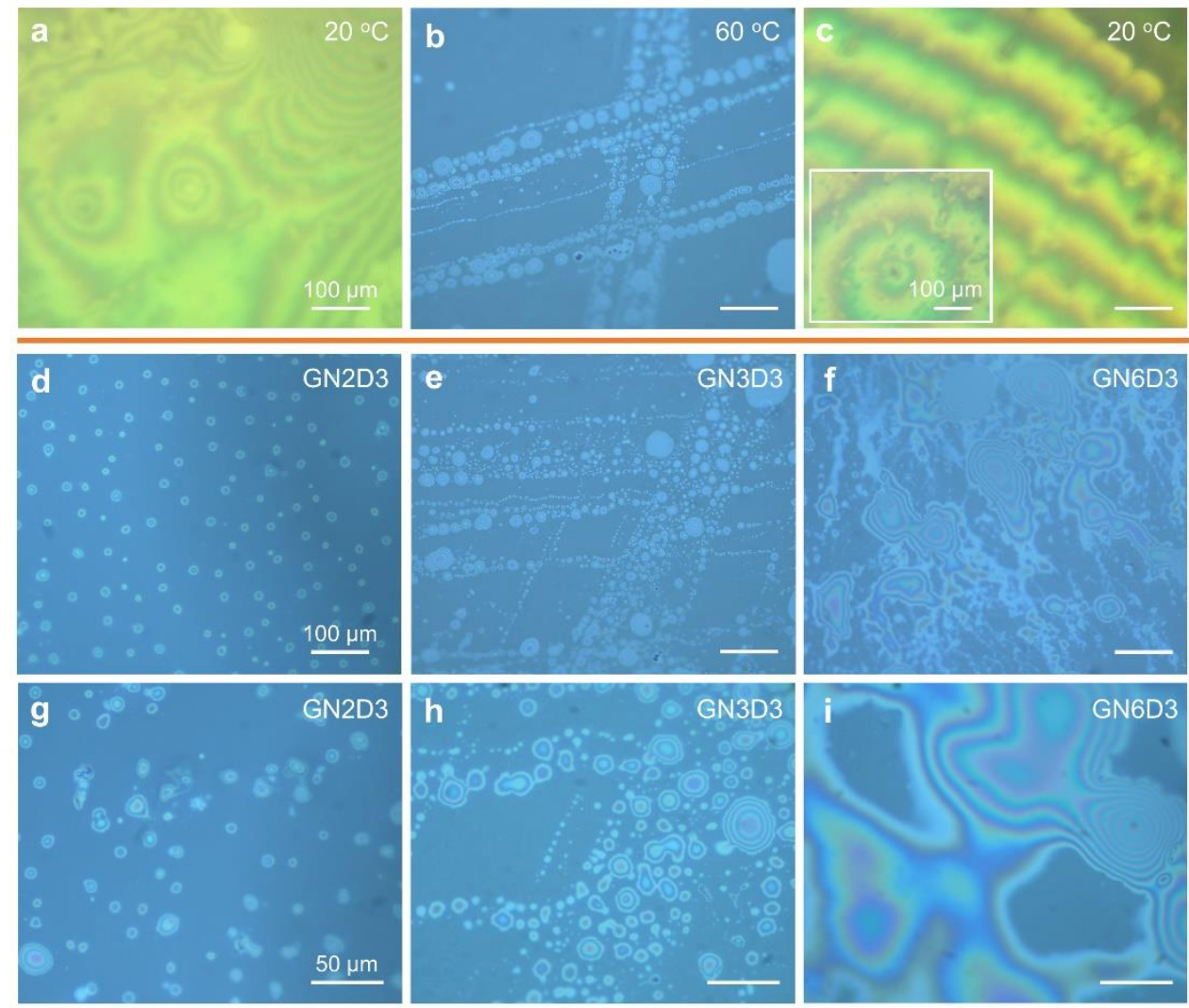

Figure 4. Visualization of microphase separation by fluorescent microscope. a-c, Fluorescent images of thermoresponsive hydrogels at preparation state (a), after immersing in water at $60{ }^{\circ} \mathrm{C}$ for $30 \mathrm{~min}(\mathbf{b})$, and after overnight recovery at room temperature (c) using GN3D3 as a representative example. $\mathbf{d}-\mathbf{i}$, Fluorescence images of microphase-separated hydrogels after immersing in water at $60{ }^{\circ} \mathrm{C}$ for 30 min, including GN2D3 (d,g), GN3D3 (e,h) and GN6D3 (f,i).

The phase-separated GN3D3 was then restored at $20{ }^{\circ} \mathrm{C}$ overnight, generating similar chromatic-yellow interference patterns with as-prepared GN3D3 (Figure 4c). Notably, an entire Newton's ring spreads across all area in the recovered pattern and possesses relatively wide fringe spacings. Such uniform pattern implies a homogenous network produced by rehydration of PNIPAM and rearrangement of PDMA/PNIPAM phases. Encouraged by the above results, the fluorescence images of GN2D3, GN3D3 and GN6D3 were then recorded to compare composition-dependent microphase separation patterns. As presented in Figure $4 \mathrm{~d}-\mathrm{i}$, the composition of PDMA/PNIPAM exerts a great influence on the microphase-separated morphologies and domain size. In GN2D3 with PDMA as the major component, uniform dispersion of PDMA-rich matrix with isolated PNIPAM-rich spherical domains is observed (Figure 4d and 4g). In GN3D3 with same composition of PNIPAM and PDMA, bicontinuous interpenetrating network is formed (Figure $4 \mathrm{e}$ and $4 \mathrm{~h}$ ). The "sea-island" pattern with isolated PDMA-rich domains and continuous PNIPAM-rich matrix appears when PNIPAM become the major component in GN6D3 (Figure 4f and 4i). In terms of Newton's rings assigned to PNIAM-rich domains, the numbers of interference fringes and the fringe spacing gradually increases from GN2D3, GN3D3 to GN6D3. This suggests that PNIPAM-rich aggregates have a larger size and more uniform assembly with increasing composition of PNIPAM. These morphological patterns demonstrate that AIE-assisted fluorescent imaging provides a powerful tool for direct visualization of stimuli-responsive morphological variation and easy differentiation of composition-dependent microphase separation in mesoscopic scale.
Mechanical properties. After understanding the stimuliresponsive and composition-dependent microphase separation of GN2D3, GN3D3 and GN6D3, we wonder the impact of such morphologies on their mechanical properties. After pre-testing the frequency-dependent viscoelastic behaviors at $20{ }^{\circ} \mathrm{C}$ and $60{ }^{\circ} \mathrm{C}$ in Figure S12, their thermoresponsive mechanical properties at small deformations were measured by rheological measurements at a constant suitable frequency. As illustrated in Figure 5a, heating the hydrogel samples from $30{ }^{\circ} \mathrm{C}$ to $40{ }^{\circ} \mathrm{C}$ triggers dramatic increase of elastic modulus ( $\left.\mathrm{G}^{\prime}\right)$ corresponding to the emergence and progress of microphase separation, where elastic actively PNIPAM network gets collapsed and stiffen. Meanwhile, G' have been enhanced with the increase of PNIPAM composition, possibly due to the conversion of PNIPAM phase from isolated domains to continuous matrix. Loss modulus ( $G$ ') ) is much lower than $G$ ' within the studied temperature range, implying the minimal contribution of dissipative losses. We then investigated the large strain behaviors of GN2D3, GN3D3 and GN6D3 at $20{ }^{\circ} \mathrm{C}$ and $60{ }^{\circ} \mathrm{C}$ by tensile stress-strain measurements (Figure S13 and S14). As shown in Figure 5b, these as-prepared hydrogel specimens behave the typical soft and brittle mechanical behaviors of hydrogels at $20{ }^{\circ} \mathrm{C}$. GN2D3 exhibit the best mechanical properties, possibly related to its highest polymer density at $20^{\circ} \mathrm{C}$. Their mechanical performance is remarkably enhanced at $60{ }^{\circ} \mathrm{C}$. The strengthened fracture strain and stress are possibly related to the conformational entanglement of collapsed PNIPAM and formation of multiple noncovalent interactions above LCST, respectively. GN6D3 shows the 

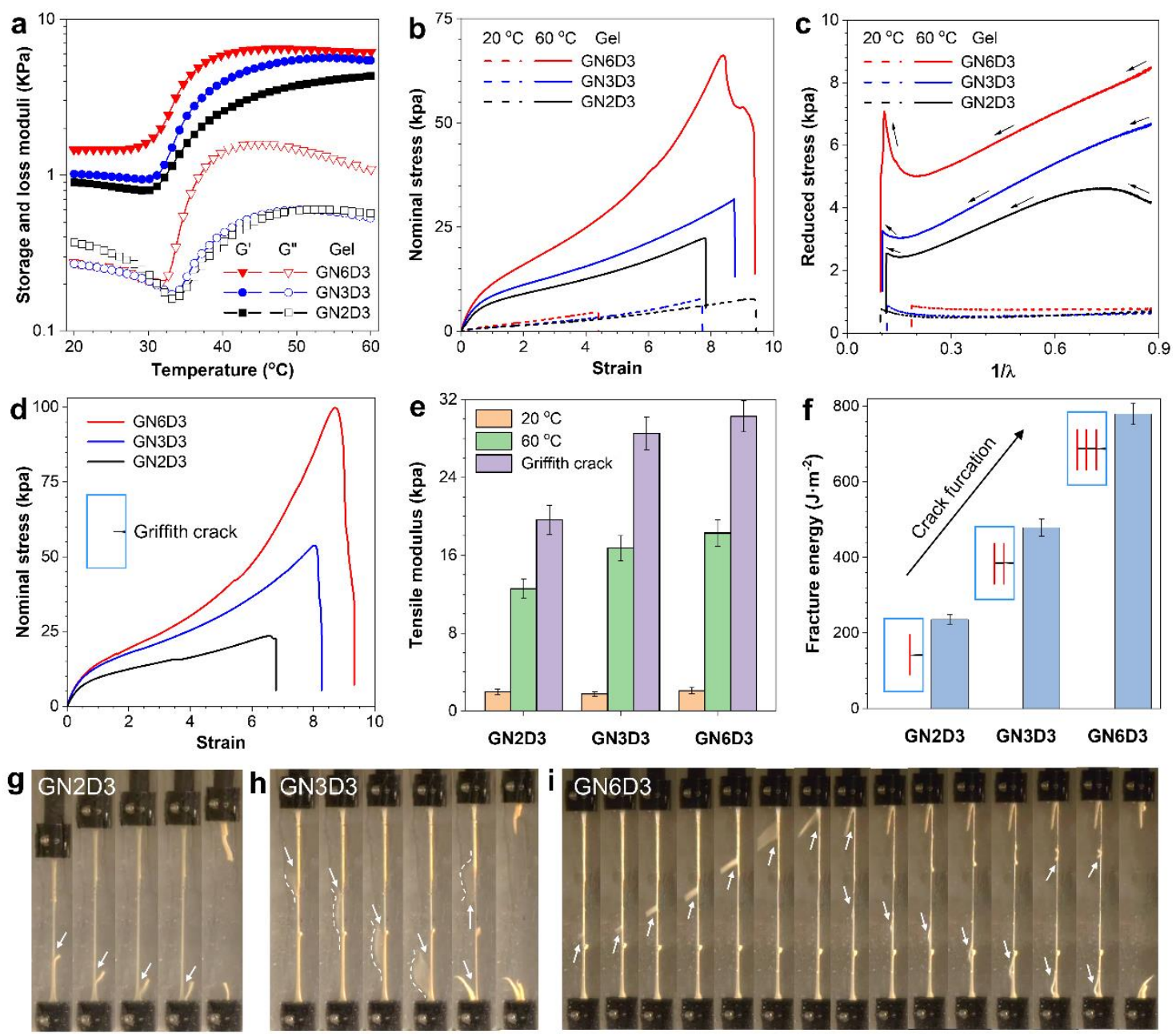

Figure 5. Thermoresponsive viscoelastic, large strain behavior and Griffith crack multifurcation. a, Temperature-dependent shear storage (solid symbols, G') and loss (open symbols, G”) moduli of GN2D3 (black), GN3D3 (blue) and GN6D3 (red) with a heating rate of $0.2^{\circ} \mathrm{C} / \mathrm{min}$. b. Tensile stressstrain curves of GN2D3, GN3D3 and GN6D3 at $20{ }^{\circ} \mathrm{C}$ and $60{ }^{\circ} \mathrm{C}$. c. Mooney-Rivlin plot of reduced stress versus $1 / \lambda$ at $20{ }^{\circ} \mathrm{C}$ and $60{ }^{\circ} \mathrm{C}$ corresponding to the stress-strain curves in (b). d, Tensile stress-strain curves of GN2D3, GN3D3 and GN6D3 with Griffith crack at $60{ }^{\circ} \mathrm{C}$. e, Tensile modulus of GN2D3, GN3D3 and GN6D3 at $20^{\circ} \mathrm{C}$ (yellow), $60{ }^{\circ} \mathrm{C}$ (green) and with Griffith crack (purple). f, Comparative analysis of fracture energies of Griffith cracked hydrogels. Inset: Schematic diagram of crack furcation referring to the actual situation. $\mathbf{g}$-i, Pictures taken during tensile tests of Griffith cracked hydrogels in paraffin oil baths at $60^{\circ} \mathrm{C}$, which record the crack propagation and multifurcation process of GN2D3 (g), GN3D3 (h) and GN6D3 (i).

best mechanical properties at $60^{\circ} \mathrm{C}$, which is consistent with the role of PNIPAM as continuous matrix in microphase separation. In addition, an interesting crack deflection occurs during the stretching process of GN6D3 (Figure S14). To graphically depict their divergence from entropy-driven elasticity, stress-strain behaviors have been plotted as reduced stress versus reciprocal elongation $(1 / \lambda)$ according to the Mooney-Rivlin semi-empirical model. ${ }^{[44]}$ As shown in Figure $5 \mathrm{c}$, the almost horizontal lines at $20{ }^{\circ} \mathrm{C}$ indicate that their original mechanical performance is dominated by entropy elasticity. In contrast, their reduced stress $-1 / \lambda$ curves at $60^{\circ} \mathrm{C}$ obviously deviate from entropic elasticity, which can be roughly separated into two stages of initial softening and final hardening. The softening behavior is contributed from the breakage of noncovalent interactions to dissipate energy during the disentanglement of collapsed PNIPAM domains. After the consumption of physical association, the polymer chains are oriented and aligned along the stretching direction, which is attributed to the hardening behavior before the fracture of chemically crosslinked polymer network. Besides, the hardening stage of GN2D3 at the early stages of deformation at $60^{\circ} \mathrm{C}$ is reasonable considering its microphase separation pattern, where the tensile stress might be transferred from the PDMA-rich matrix to the isolated PNIPAM-rich domains.

Inspired by the crack deflection in GN6D3, tensile tests of hydrogels with Griffith crack at $60{ }^{\circ} \mathrm{C}$ were measured to study the crack propagation path by introducing the high stress concentration zone at crack tip. Their tensile stress-strain curves are plotted in Figure $5 \mathrm{~d}$ and their corresponding stretching process are recorded by pictures (Figure $5 \mathrm{~g}-\mathrm{i}$ ) and videos (Videos S1-3). In general, hydrogel specimens with Griffith crack behave further reinforced mechanical properties than normal tensile specimens, owing to the contribution of crack furcation as additional energy dissipation pathway. GN2D3 with Griffith crack displays the blunting and one-way deflection along the stretching direction after crack propagation for a short while (Figure $5 \mathrm{~g}$ ), resulting in relatively decreasing fracture surface area and fracture stress. As depicted in Figure 5h and 5i, GN3D3 performs crack trifurcation from the crack tip and various edges of stretching specimens, and GN6D3 is further developed into crack multifurcation. Both GN3D3 and GN6D3 with Griffith crack demonstrate the evidently enhanced fracture stress than normal specimens under comparative fracture strain. In fact, theoretical criterion for crack deflection has been expressed as the adhesive energy of the interface is less than the cohesive 
energy in a certain ratio. ${ }^{[45]}$ As the composition of PNIPAM increases, the cohesive energy of hydrogels is intensified and the possibility of encountering the PNIPAM/PDMA interfaces on the crack path also raises. This explains the increasing crack multifurcation from GN2D3, GN3D3 to GN6D3. Indicated by comparative analysis of the tensile moduli in Figure 5e, evident enhancement at $60{ }^{\circ} \mathrm{C}$ is related to the strengthened PNIPAM domains and further improvement with Griffith crack possibly originates from the initial orientation and alignment at crack tip. The fracture energy of specimens with Griffith crack, describing the strain energy required to propagate the crack across hydrogels, has been calculated in Figure 5f. GN6D3 with crack multifurcation displays highest fracture energy at around 800 $\mathrm{J} / \mathrm{m}^{2}$ partially related to its largest fracture surface area, which resembles with the fiber-assembled biological tissues. Acquiring such ability to resist crack penetration and maintain the integrity of the entire by partial sacrifice in strengthened hydrogels is of great significance for material engineering and practical applications.

Mechanism study. Based on the above studies on microscopic hydrophilicity-hydrophobicity transformation, mesoscopic morphological variation and macroscopic mechanical performance, we propose a strengthening mechanism as illustrated in Figure 6. So far, most scientist has associated the LCST behavior of PNIPAM with the hydrophobic interactions existed in the hydrophobic backbone and isopropyl groups ${ }^{[46]}$, but this is not all. According to the microenvironmental polarity changes indicated by TICT-active AIEgen, it is assumed that thermoresponsive PNIPAM network has completely transformed from hydrophilicity to hydrophobicity (Figure 6a). By analyzing the molecular structure of PNIPAM, hydrogen bond acceptors provide accessibility to water molecules and thus the extended hydrated PNIPAM chains are water-soluble. However, the coil-to-globule transition at LCST generates multiple intra/interchain hydrogen bonds in the collapsed PNIPAM, which will occupy the previous hydrogen bond accepters for water and result in waterinsolubility of whole PNIPAM domains. In this way, the PNIPAM domains are strengthened significantly due to the massive generation of noncovalent interactions, mainly hydrogen bonds. Such strengthened hydrogels gradually break noncovalent interactions to dissipate strain energy during the disentanglement of collapsed PNIPAM domains (Figure 6b). The orientation and alignment of polymer network with regained noncovalent interactions are induced in highly stretched hydrogels, leading to reinforced performance in the final stage before fracture. Hence, the mechanical performance of PNIPAM/PDMA composite hydrogels is improved by simple heating process when the macroscopic volume change is balanced by the further hydration of PDMA domains. The role of microphase separation in reinforcing hydrogels is well demonstrated in
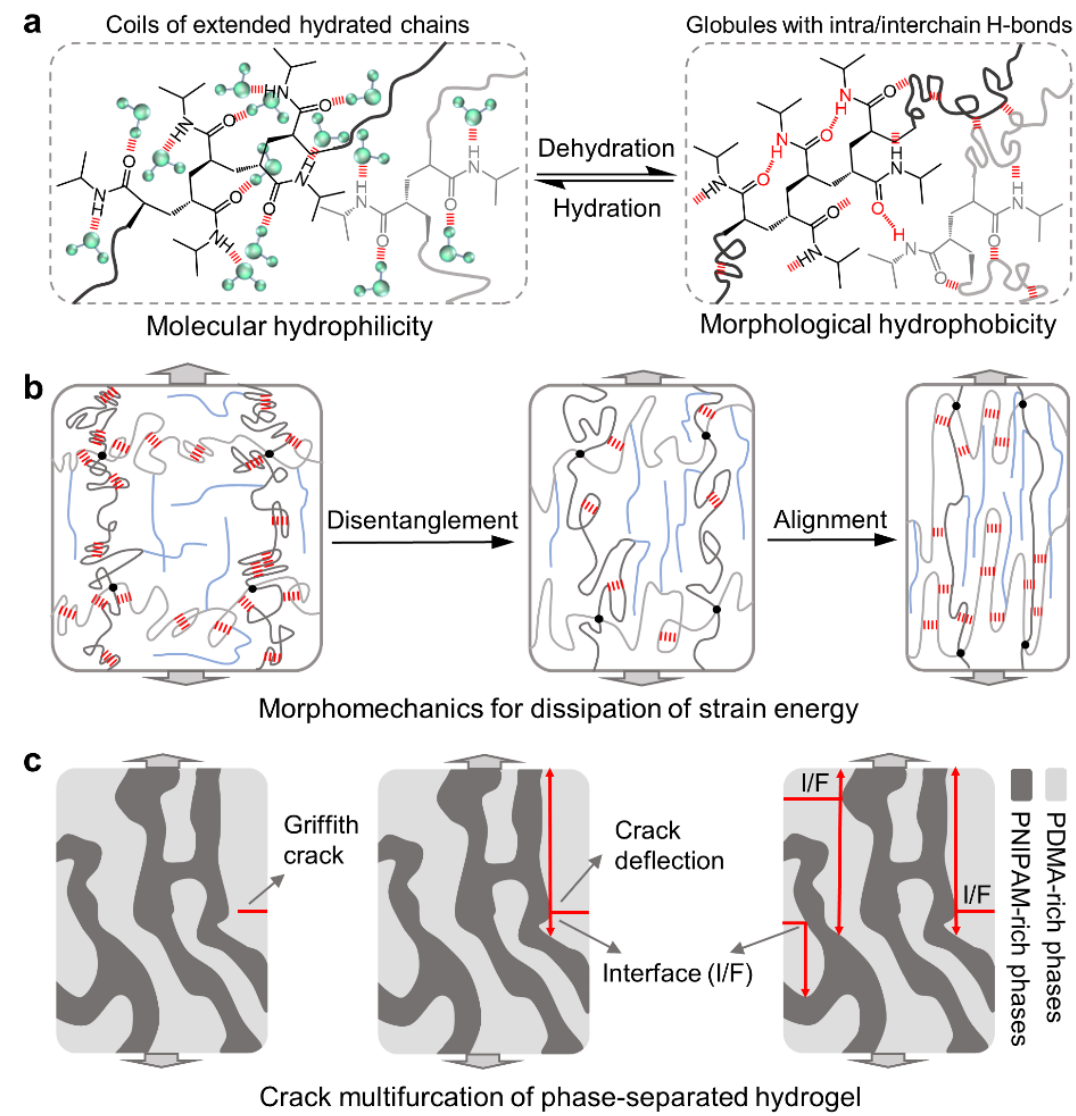

Figure 6. Proposed mechanism for thermoresponsive toughing and Griffith crack multifurcation. a, The reversible transformation of molecular hydrophilicity and morphological hydrophobicity during thermally induced hydration and dehydration of PNIPAM network. The previous hydrogen bond accepters in PNIPAM for water molecules are occupied by intra/interchain hydrogen bonds above LCST. b, Consumption of noncovalent interactions, mainly hydrogen bonds, contributes to the strain energy dissipation during the disentanglement of collapsed PNIPAM network. The regained noncovalent interactions during the orientation and alignment of the highly stretched PNIPAM network reinforce tensile strength again before fracture. c, Proposed multifurcation mechanism of microphase separated hydrogels introduced by Griffith crack. Griffith crack meets the weak interface of PDMA-rich (light grey) and PNIPAM-rich (dark grey) phases and induces crack deflection along the stretching direction. Increasing fracture surface area by crack multifurcation further retards the fracture failure and enlarges fracture energy. 
the tensile measurements with Griffith crack. As shown in Figure $6 \mathrm{c}$, the crack propagation along the horizontal direction is blocked when the crack tip meets the weak interfaces between PNIPAM and PDMA phases. Since the adhesive energy of PNIPAM/PDMA interfaces is incomparable to the cohesive energy, the crack deflects along the vertical stretching direction. Meanwhile, the concentrated tensile stress activates the chain orientation and alignment around the initial crack tip. This helps generate more notches at the edge of hydrogel, perhaps in the weak PDMA-rich domains. In other words, the heterogeneous distribution of strong PNIPAM phases and weak PDMA phases promotes the formation of crack multifurcation and thus enlarges fracture energy. Furthermore, the composition-dependent crack multifurcation is corresponding to their cohesive energy and the possibility of meeting PNIPAM/PDMA interfaces, which is predictable from their microphase separation patterns. Briefly, the above-mentioned mechanical behaviors of sacrificing the noncovalent interactions or weak interfaces between two separated phases to retard the fracture failure and preserve the integrity of the whole, exactly match the proposed concept of morphomechanics.

In summary, we have successfully realized the direct visualization of microphase separation and easy differentiation of hydrophobic collapsed PNIPAM domains in the PNIPAM/PDMA hydrogels by using AIEgen as a fluorescent indicator. The composition-dependent microphase separation can be readily identified as spherical domains, bicontinuous network and "sea-island" patterns in a facile, high-contrast and noninvasive manner. In contrast to the general understanding of "hydrophobic interactions", the transformation from molecular hydrophilicity to morphological hydrophobicity has been attributed to the formation of multiple inter/intrachain hydrogen bonds. As the universality of this transformation has been proved by several stimuli-responsive sol-gel transitions, such conceptual revolution will have a great impact on the material research and biological study. For example, it can help us understand how biopolymers regulate their inter/intra-assemblies via phase transition to perform various biological functions such as reactions acceleration, signal transduction and transcriptional regulation. ${ }^{[4]}$

The formation of multiple non-covalent interactions and microphase separation also contribute to the thermoreponsive mechanical strength and fracture resistance by crack deflection. The composition-dependent crack multifurcation has been associated with microphase separation patterns, which provides an additional energy dissipation pathway and enhances fracture energy. Such behavior actually mimics the tearing of tissues along the weak interfaces between highstrength fibers. Overall, the mechanism study has been well summarized by the proposed concept of morphomechanics. We hope that this work can serve as an inspiration for the mesoscopic exploration of stimuli-responsive materials and contribute to the development of high-strength hydrogels and biomimetic materials.

\section{Supporting Information}

The Supporting Information is available free of charge.

\section{Conflict of Interest}

The authors declare no conflict of interest.

\section{Acknowledgments}

We are grateful for the financial support from the National Science Foundation of China (21788102, 21490570, and 21490574), the Research Grant Council of Hong Kong (16305618, 16304819, N-HKUST609/19, and C6009-17G), the Science and Technology Plan of Shenzhen (JCYJ20160229205601482， JCYJ20170818113602462, JCYJ20180306180231853, and JCYJ 20180306174910791), the Innovation and Technology Commission (ITCCNERC14SC01), and National Key Research and Development Program of China (2018YFE0190200). Y.B.H. thanks Prof. Dominique Hourdet and Prof. Nicolas Sanson for their kind discussions and suggestions during her research exchange in ESPCI ParisTech. Y.B.H. also thanks Xiaolin Liu, Louis Debertrand and Zuxiang $\mathrm{Xu}$ for their technical assistance.

\section{References}

[1] K. Y. Lee, D. J. Mooney, Chem. Rev. 2001, 101, 1869.

[2] T. R. Hoare, D. S. Kohane, Polymer 2008, 49, 1993.

[3] J. Li, D. J. Mooney, Nat. Rev. Mater. 2016, $1,1$.

[4] P. H. Kouwer, M. Koepf, V. A. Le Sage, M. Jaspers, A. M. van Buul, Z. H. Eksteen-Akeroyd, T. Woltinge, E. Schwartz, H. J. Kitto, R. Hoogenboom, Nature 2013, 493, 651. [5] Z. Lei, Q. Wang, S. Sun, W. Zhu, P. Wu, Adv. Mater. 2017 29, 1700321.

[6] K. Deligkaris, T. S. Tadele, W. Olthuis, A. van den Berg, Sens. Actuators, B 2010, 147, 765.

[7] S. I. Park, D. S. Brenner, G. Shin, C. D. Morgan, B. A. Copits, H. U. Chung, M. Y. Pullen, K. N. Noh, S. Davidson, S. J. Oh, Nat. Biotechnol. 2015, 33, 1280.

[8] S. A. Morin, R. F. Shepherd, S. W. Kwok, A. A. Stokes, A. Nemiroski, G. M. Whitesides, Science 2012, 337, 828.

[9] M. Wehner, R. L. Truby, D. J. Fitzgerald, B. Mosadegh, G. M. Whitesides, J. A. Lewis, R. J. Wood, Nature 2016, 536, 451.

[10] L. Dong, A. K. Agarwal, D. J. Beebe, H. Jiang, Nature 2006, 442, 551 .

[11] J. Guo, X. Liu, N. Jiang, A. K. Yetisen, H. Yuk, C. Yang, A. Khademhosseini, X. Zhao, S. H. Yun, Adv. Mater. 2016, 28, 10244.

[12] C. Yang, Z. Suo, Nat. Rev. Mater. 2018, 3, 125.

[13] J. A. Rogers, T. Someya, Y. Huang, Science 2010, 327, 1603.

[14] H. Yuk, B. Lu, X. Zhao, Chem. Soc. Rev. 2019, 48, 1642. [15] U. G. Wegst, H. Bai, E. Saiz, A. P. Tomsia, R. O. Ritchie, Nat. Mater. 2015, 14, 23.

[16] W. Wang, Y. Zhang, W. Liu, Prog. Polym. Sci. 2017, 71, 1.

[17] T. Scheibel, Curr. Opin. Biotechnol. 2005, 16, 427.

[18] M. J. Buehler, S. Keten, Nano Res. 2008, 1, 63.

[19] C. Riekel, C. Craig, M. Burghammer, M. Müller, Naturwissenschaften 2001, 88, 67.

[20] J. E. Muscolino, Kinesiology-E-Book: The Skeletal System and Muscle Function. Elsevier Health Sciences 2014, p. 49 .

[21] R. Bahr, S. Mæhlum, Clinical guide to sports injuries. Human Kinetics 2004, p. 7.

[22] C. W. Peak, J. J. Wilker, G. Schmidt, Colloid. Polym. Sci. 2013, 291, 2031.

[23] M. Shibayama, Soft Matter 2012, 8, 8030. 
[24] J. P. Gong, Y. Katsuyama, T. Kurokawa, Y. Osada, Adv. Mater. 2003, 15, 1155.

[25] Y. Okumura, K. Ito, Adv. Mater. 2001, 13, 485.

[26] K. Haraguchi, T. Takehisa, Adv. Mater. 2002, 14, 1120.

[27] T. Sakai, T. Matsunaga, Y. Yamamoto, C. Ito, R. Yoshida, S. Suzuki, N. Sasaki, M. Shibayama, U. i. Chung, Macromolecules 2008, 41, 5379.

[28] K. J. Henderson, T. C. Zhou, K. J. Otim, K. R. Shull, Macromolecules 2010, 43, 6193.

[29] J. Kopeček, Biomaterials 2007, 28, 5185.

[30] Q. Chen, H. Chen, L. Zhu, J. Zheng, J. Mater. Chem. B 2015, 3, 3654 .

[31] B. Wu, W. Chassé, R. Peters, T. Brooijmans, A. A. Dias, A. Heise, C. J. Duxbury, A. P. Kentgens, D. F. Brougham, V. M. Litvinov, Macromolecules 2016, 49, 6531.

[32] E. Ducrot, Y. Chen, M. Bulters, R. P. Sijbesma, C. Creton, Science 2014, 344, 186.

[33] Y. Tanaka, EPL 2007, 78, 56005.

[34] C. Creton, M. Ciccotti, Rep. Prog. Phys. 2016, 79, 046601.

[35] J. S. Mohammed, W. L. Murphy, Adv. Mater. 2009, 21, 2361.
[36] S. L. James, C. J. Adams, C. Bolm, D. Braga, P. Collier, T. Friščić, F. Grepioni, K. D. Harris, G. Hyett, W. Jones, Chem. Soc. Rev. 2012, 41, 413.

[37] T. Förster, K. Kasper, Z. Elektrochem, Ber. Bunsenges. Phys. Chem. 1955, 59, 976.

[38] J. Mei, N. L. Leung, R. T. Kwok, J. W. Lam, B. Z. Tang, Chem. Rev. 2015, 115, 11718.

[39] R. Hu, E. Lager, A. Aguilar-Aguilar, J. Liu, J. W. Lam, H. H. Sung, I. D. Williams, Y. Zhong, K. S. Wong, E. PenaCabrera, J. Phys. Chem. C 2009, 113, 15845.

[40] K. Sato, T. Nakajima, T. Hisamatsu, T. Nonoyama, T. Kurokawa, J. P. Gong, Adv. Mater. 2015, 27, 6990.

[41] H. Guo, N. Sanson, D. Hourdet, A. Marcellan, Adv. Mater. 2016, 28, 5857.

[42] T. Nonoyama, Y. W. Lee, K. Ota, K. Fujioka, W. Hong, J. P. Gong, Adv. Mater. 2019, 1905878.

[43] M. Heskins, J. E. Guillet, J. Macromol. Sci. A. 1968, 2, 1441.

[44] M. Mooney, J. Appl. Phys. 1940, 11, 582.

[45] K. Kendall, P. Roy. Soc. A-math. Phy. 1975, 344, 287.

[46] C. Wu, S. Zhou, Macromolecules 1995, 28, 8381.

[47] E. Dolgin, Nature 2018, 555.

\section{The table of contents entry:}

The direct visualization of microphase separation and easy differentiation of hydrophilicity-hydrophobicity transformation has been achieved in a facile, high-contrast and noninvasive manner by using luminogens with aggregation-induced emission as fluorescent indicators. Based on the morphological observation and mechanical study, the concept of morphomechanics with a comprehensive mechanism clarification is proposed.

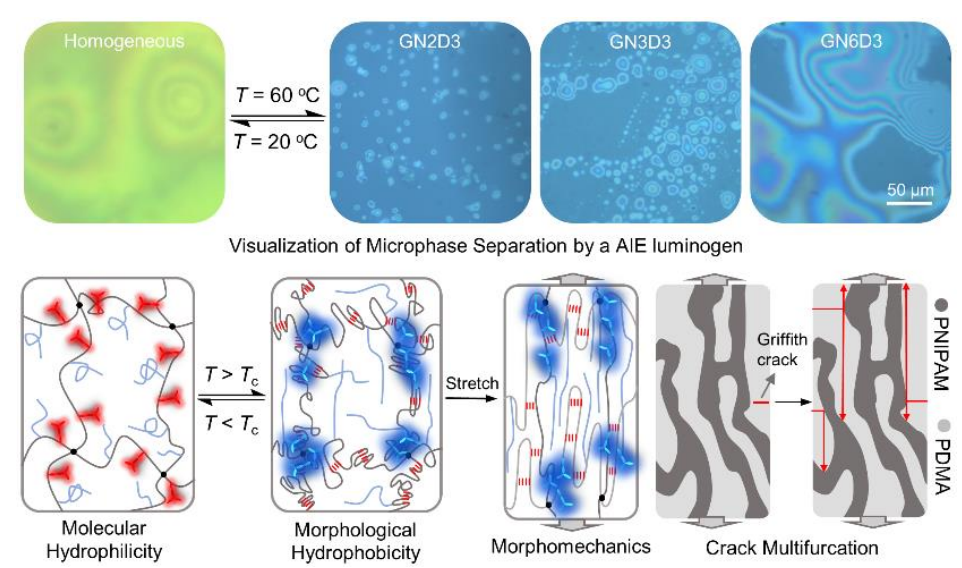

Table of Contents 


\section{Making Hydrogels Stronger through Hydrophilicity-Hydrophobicity Transformation, Thermoresponsive Morphomechanics and Crack Multifurcation}

Yubing $\mathrm{Hu}^{1,2}$, Lucile Barbier ${ }^{3}$, Zhao $\mathrm{Li}^{1,2}$, Xiaofan $\mathrm{Ji}^{1,2}$, Heiva Le Blay ${ }^{3}$, Junkai Liu ${ }^{1,2}$, Jacky W. Y. Lam ${ }^{1,2, *}$, Alba Marcellan ${ }^{3, *}$, and Ben Zhong Tang ${ }^{1,2,4, *}$

\section{Supporting Information}

Materials. All the chemicals were reagent grade and used as received without further purification. 4-bromo-N,N-diphenylaniline, 4-vinylpyridine, 2-bromoethanol, acryloyl chloride, palladium(II) acetate, tri(o-tolyl)phosphine, sodium sulfate, $N$-isopropylacrylamide (NIPAM), $N, N$-dimethylacrylamide (DMA), $N, N^{\prime}$-methylenebisacrylamide (MBA), potassium peroxodisulfate (KPS), $N, N, N^{\prime}, N^{\prime}$-tetramethylethylenediamine (TEMED), 2-aminoethanethiol hydrochloride, acetic acid, sodium hydroxide, acrylic acid and $N, N^{\prime}$-dicyclohexylcarbodiimide were purchased from Sigma-Aldrich and J\&K Scientific. PS $\left(M_{\mathrm{w}}=280,000 \mathrm{~g} / \mathrm{mol}\right), \operatorname{PB}\left(M_{\mathrm{w}}=\right.$ $200,000 \mathrm{~g} / \mathrm{mol})$, PMMA $\left(M_{\mathrm{w}}=120,000 \mathrm{~g} / \mathrm{mol}\right)$, PEG $\left(M_{\mathrm{w}}=20,000 \mathrm{~g} / \mathrm{mol}\right)$, chitosan $(85 \%$ deacetylated), PEG $\left(M_{\mathrm{w}}=6,000 \mathrm{~g} / \mathrm{mol}\right), \alpha$-cyclodextrin, Pluronic ${ }^{\circledR}$ F-127 (PEG-PPG-PEG, $M_{\mathrm{w}}$ $=12,600 \mathrm{~g} / \mathrm{mol}$ ) and Mowiol ${ }^{\circledR}$ PVA-117 (Poly(vinyl alcohol), $M_{\mathrm{w}}=145,000 \mathrm{~g} / \mathrm{mol}$ ) were purchased from Sigma-Aldrich and Meryer. All the organic solvents including toluene, acetone, dimethyl sulfoxide (DMSO) and methanol $(\mathrm{MeOH})$, triethylamine, dichloromethane (DCM), hexane, ethyl acetate, triethylamine (TEA), dimethylformamide (DMF) and N-methyl-2pyrrolidone (NMP) were purchased from Sigma-Aldrich. Water was purified with a Millipore system with inverse osmosis membrane (Milli RO) and ion exchange resins (Milli Q).

Instrumentation. ${ }^{1} \mathrm{H}$ and ${ }^{13} \mathrm{C}$ NMR spectra were obtained on a Bruker ARX 400 NMR spectrometer in deuterated dimethyl sulfoxide with tetramethylsilane (TMS; $\delta=0 \mathrm{ppm}$ ) as internal reference. High-resolution mass spectra (HRMS) were recorded on a GCT premier CAB048 mass spectrometer operating in MALDI-TOF mode. Gel permeation chromatography (GPC) was performed in water at an elution rate of $1.0 \mathrm{~mL} / \mathrm{min}$ on a Waters Associates GPC system equipped with a Waters 1515 HPLC pump and interferometric refractometer detector. Standard poly(methyl acrylate)s were utilized for ploting calibration curves for number-average molecular weights $\left(M_{\mathrm{w}}\right)$ and weight-average molecular weight $\left(M_{\mathrm{w}}\right)$ determination. The polymer samples were dissolved in $0.02 \mathrm{M}$ sodium hydroxide $(2 \mathrm{mg} / \mathrm{mL})$ filtered through a $0.45 \mu \mathrm{m}$ PTFE filter and then injected into the GPC system. The refractive 
index of the hydrogels were determined using an Alpha-SE Woollam ellipsometer. UV-vis spectra were collected on a Hewlett-Packard 8453 spectrophotometer equipped with a temperature controller $\left( \pm 0.1^{\circ} \mathrm{C}\right)$. Transmittance curves were built by collecting the absorbance at $600 \mathrm{~nm}$ with a scanning rate of $0.3{ }^{\circ} \mathrm{C} / \mathrm{min}$. Photoluminescence (PL) spectra were measured on a Horiba Fluorolog spectrophotometer. Fluorescence quantum yields were measured using a Horiba Quanta- $\varphi$ accessory combined with a Horiba fluorometer. Photoluminescence images were taken by Olympus BX41 fluorescent microscope with SPOT RT3 mono charge-coupled device (CCD) camera under ultraviolet light excitation $(330-385 \mathrm{~nm})$.

\section{Synthesis and Characterization.}

a)

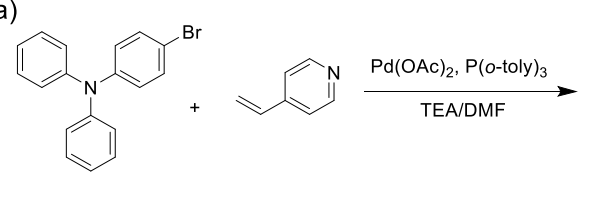

b)
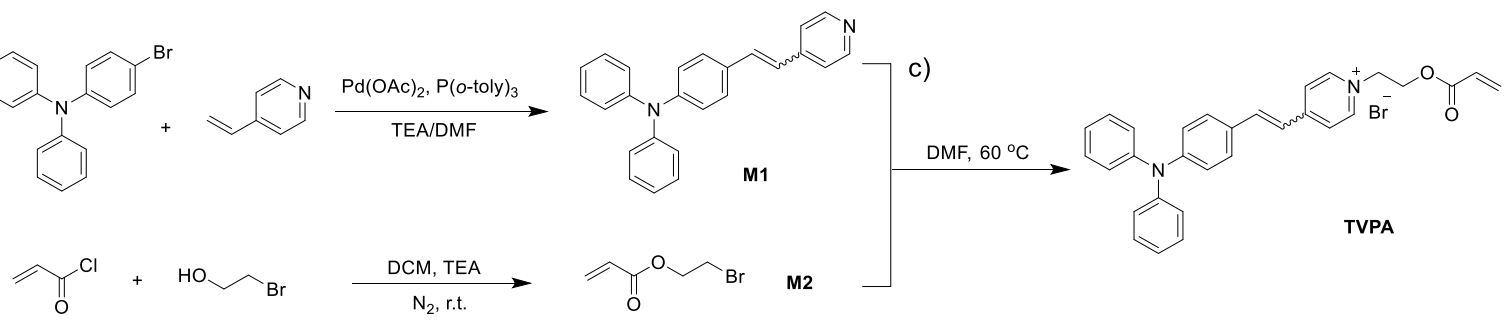

Figure S1. Three-step synthetic routes to TVPA.

Synthesis of M1: A typical procedure for Heck cross-coupling reaction was conducted to synthesis $\mathbf{M 1}$ by the starting materials of 4-bromo-N,N-diphenylaniline (2.53 g, $7.8 \mathrm{mmol}$ ) and 4-vinylpyridine (1.07 g, $1.02 \mathrm{mmol})$. The crude product was purified by column chromatography (SiO2; DCM/hexane, 1:1). Yellow solid (1.67 g, $59 \%$ yield). ${ }^{1} \mathrm{H}$ NMR $\left(\mathrm{CDCl}_{3}\right): \delta=6.94-6.90(\mathrm{~d}, 1 \mathrm{H}), 7.12-7.08(\mathrm{~m}, 4 \mathrm{H}), 7.17-7.15(\mathrm{~d}, 4 \mathrm{H}), 7.33-7.30(\mathrm{~m}, 7 \mathrm{H})$, 7.37-7.36 (d, 2H), 8.58-8.57 (d, 2H) ppm. ${ }^{13} \mathrm{C}$ NMR $\left(\mathrm{CDCl}_{3}\right): \delta=120.67,122.85,123.54$, 124.93, 128.02, 129.44, 129.83, 132.70, 144.99, 147.30, 148.47, 150.17 ppm. HRMS (MALDI) $\mathrm{m} / \mathrm{z}$ calcd. for $\mathrm{C}_{25} \mathrm{H}_{20} \mathrm{~N}_{2}: 348.1626\left[\mathrm{M}+\mathrm{H}^{+}\right]$, found 348.1750 .

Synthesis of M2. M2 was synthesized according to previously reported procedures. ${ }^{1} 2$ bromoethanol (1.24 g, $10 \mathrm{mmol})$ was added into a two-necked flask and refilled with nitrogen for three times. DCM $(30 \mathrm{~mL})$ was added and the triethylamine $(1.4 \mathrm{~mL})$ was injected at $0{ }^{\circ} \mathrm{C}$. Acryloyl chloride $(0.90 \mathrm{~g}, 10 \mathrm{mmol})$ in DCM $(2 \mathrm{~mL})$ was added dropwise and the reaction mixture was stirred for overnight at room temperature. Upon completion, the reaction mixture was filtered and the filtrate was washed with DCM/water for three times. The organic layer 
was dried over anhydrous $\mathrm{Na}_{2} \mathrm{SO}_{4}$ and went rotary evaporation and vacuum to get the product (1.52 g, 85\% yield, colorless liquid). ${ }^{1} \mathrm{H}$ NMR $\left(\mathrm{CDCl}_{3}\right): \delta=6.44$ (dd, $\left.1 \mathrm{H}\right), 6.14-6.10$ (dd, 1 $\mathrm{H}), 5.85(\mathrm{dd}, 1.4 \mathrm{~Hz}, 1 \mathrm{H}), 4.42(\mathrm{t}, 2 \mathrm{H}), 3.51(\mathrm{t}, 2 \mathrm{H}) \mathrm{ppm} .{ }^{13} \mathrm{C} \mathrm{NMR}\left(\mathrm{CDCl}_{3}\right): \delta=165.52$, $131.65,127.81,63.82,28.59$.

Synthesis of TVPA. M1 (1.45 g, $4 \mathrm{mmol})$ and M2 $(0.86 \mathrm{~g}, 4.8 \mathrm{mmol})$ were dissolved in the $\mathrm{DMF}$ and the reaction mixture was stirred for overnight at $80^{\circ} \mathrm{C}$. Upon completion, the reaction mixture was filtered and the filtrate was washed with DCM/water for three times. The organic layer was dried over anhydrous $\mathrm{Na}_{2} \mathrm{SO}_{4}$ and dried by rotary evaporation. The crude product was purified by column chromatography (SiO2; DCM/ethyl acetate, 1:1). Red solid (1.45 g, $69 \%$ yield). ${ }^{1} \mathrm{H}$ NMR (DMSO- $\left.d_{6}\right): \delta=4.60(\mathrm{t}, 2 \mathrm{H}), 4.81$ (t, 2H), 6.00 (d, $\left.1 \mathrm{H}\right), 6.136 .17$ (m, 1 H), 6.30-6.35 (d, 1H), $6.95(\mathrm{~d}, 2 \mathrm{H}), 7.11-7.18(\mathrm{~m}, 6 \mathrm{H}), 7.36-7.40(\mathrm{~m}, 5 \mathrm{H}), 7.62(\mathrm{~d}, 2 \mathrm{H}), 7.97$ $(\mathrm{d}, 1 \mathrm{H}), 8.17$ (t, $2 \mathrm{H}), 8.89$ (d, 2H) ppm. ${ }^{13} \mathrm{C}$ NMR (DMSO- $\left.d_{6}\right): \delta=58.05,62.63,120.34,120.49$, $120.58,122.90,123.01,124.37,125.25,127.46,127.91,129.71,129.75,132.38,141.16$, 146.06, 149.45, 153.74, 164.73. HRMS (MALDI) m/z calcd. for $\mathrm{C}_{30} \mathrm{H}_{27} \mathrm{BrN}_{2} \mathrm{O}_{2}: 526.1256$ [M+ $\mathrm{H}+]$, found 527.1318 .

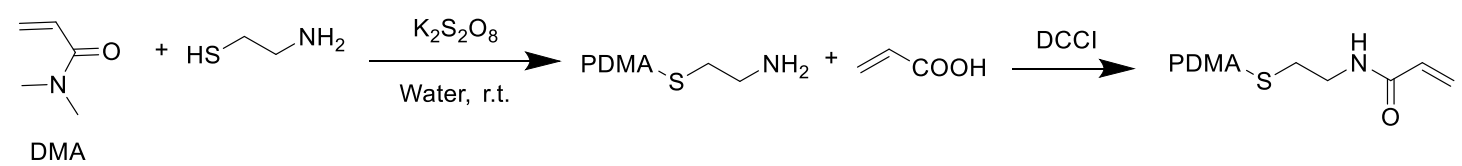

Figure S2. Synthetic routes to PDMA macromonomers.

\section{Synthesis of PDMA macromonomers.}

Synthesis of Amino-Terminated Telomers. Radical polymerization by the chain transfer agent 2-Aminoethanethiol hydrochloride $(\mathrm{AET} \cdot \mathrm{HCl})$ was conducted to produce the functional polymer chains. In a two-necked flask, 0.2 mol of monomers (DMA) was dissolved in $500 \mathrm{~mL}$ of water and the mixture was deoxygenated by nitrogen bubbling for $1 \mathrm{~h}$. The redox initiators, KPS $(4.6 \mathrm{mmol})$ and $\mathrm{AET} \cdot \mathrm{HCl}(3.1 \mathrm{mmol})$, were separately dissolved in $10 \mathrm{~mL}$ of water and deoxygenated for $30 \mathrm{~min}$ before adding into the reaction medium. The reaction mixture was stirred for overnight in an ice bath. $2 \mathrm{~mL}$ sodium hydroxide $(2 \mathrm{M})$ was added to neutralize the hydrochloride ions and the polymer solution was freeze-dried to get the telomers $(10.8 \mathrm{~g})$. 
Synthesis of PDMA macromonomers. $10 \mathrm{~g}$ of amino-terminated telomers and acrylic acid $(2.23 \mathrm{~g}, 31 \mathrm{mmol})$ were dissolved in $100 \mathrm{~mL}$ of NMP. N,N'-Dicyclohexylcarbodiimide $(3.19 \mathrm{~g}$, $155 \mathrm{mmol}$ ) was dissolved in $5 \mathrm{~mL}$ of NMP and then injected into the reaction mixture. The reaction was stirred overnight at room temperature. After dilution with $200 \mathrm{~mL}$ of water, the polymer was purified by dialysis against pure water (membrane cut-off $=12 \mathrm{kDa}$ ) for one week, and the aqueous solutions were filtered and freeze dried. The PDMA macromonomer was obtained for further hydrogel synthesis $\left(9.6 \mathrm{~g}, M_{\mathrm{n}}=240,073, M_{\mathrm{w}} / M_{\mathrm{n}}=1.6\right)$.

Table S1. Hydrogel topologies for preparation hydrogels

\begin{tabular}{llcccccccc}
\hline Sample & $\begin{array}{c}\text { Water } \\
(\mathrm{mL})\end{array}$ & $\begin{array}{c}\text { NIPAM } \\
(\mathrm{g})\end{array}$ & $\begin{array}{c}\text { PDMA } \\
(\mathrm{g})\end{array}$ & $\begin{array}{c}\text { TVPA } \\
(\%)\end{array}$ & $\begin{array}{c}\text { MBA } \\
(\%)\end{array}$ & $\begin{array}{c}\text { KPS/TEMED } \\
(\%)\end{array}$ & $\mathrm{Q}_{0}{ }^{\mathrm{a}}$ & $\mathrm{Q}_{20}{ }^{\circ} \mathrm{C}^{\mathrm{b}}$ & $\mathrm{Q}_{600^{\circ} \mathrm{C}^{\mathrm{b}}}$ \\
\hline GN & 4 & 0.3981 & $/$ & 0.05 & 0.2 & 0.5 & 11 & $48.1 \pm 5.2$ & $1.9 \pm 0.2$ \\
GD & 4 & $/$ & $0.3981^{\mathrm{c}}$ & 0.05 & 0.2 & 0.5 & 11 & $1069 \pm 62$ & $981 \pm 78$ \\
GN2D3 & 3.8 & 0.3981 & 0.5973 & 0.05 & 0.2 & 0.5 & 4.8 & $62.8 \pm 4.6$ & $8.1 \pm 0.7$ \\
GN3D3 & 4 & 0.3981 & 0.3981 & 0.05 & 0.2 & 0.5 & 6.0 & $51.6 \pm 3.7$ & $6.2 \pm 0.5$ \\
GN6D3 & 4.2 & 0.3981 & 0.1991 & 0.05 & 0.2 & 0.5 & 8.0 & $46.8 \pm 4.9$ & $3.3 \pm 0.4$ \\
\hline
\end{tabular}

${ }^{\mathrm{a}} \mathrm{Q}_{0}$ refers to the swelling ratio of preparation hydrogels. ${ }^{\mathrm{b}} \mathrm{Q}_{20^{\circ} \mathrm{C}}$ and $\mathrm{Q}_{60^{\circ} \mathrm{C}}$ refers to the swelling degree of hydrogels in thermodynamic equilibrium at $20{ }^{\circ} \mathrm{C}$ and $60{ }^{\circ} \mathrm{C}$, respectively. ${ }^{\circ} \mathrm{GD}$ was prepared using 0.3981 g DMA.

Preparation of hydrogels. Monomer (NIPAM, DMA and TVPA), cross-linker (stock solution of MBA) and initiator (stock solution of KPS) were initially dissolved in water. The degassing of reaction mixture was carried out by nitrogen bubbling in ice bath for $30 \mathrm{~min}$. Then all the solutions were mixed and TEMED was added into the reaction medium. After fast mixing (30 s), the final formulation was rapidly transferred between glass plates of $2 \mathrm{~mm}$ width under a nitrogen atmosphere, and the reaction was left to proceed overnight in the room temperature to get the as-prepared hydrogel. The cross-linker molar ratio, the initiator ratio and the density of elastically active chains in all hydrogels was controlled as same.

Swelling measurement: Equilibrium swelling experiments were performed in pure water at designated temperatures. The preparation hydrogel samples, were initially cut and placed in a large excess of water for one week. The swollen hydrogels were weighed $\left(\mathrm{m}_{\mathrm{e}}\right)$ at equilibrium 
and the swelling degree $(\mathrm{Q})$ were calculated as $\mathrm{Q}=\mathrm{m}_{\mathrm{e}} / \mathrm{m}_{\mathrm{d}}, \mathrm{m}_{\mathrm{d}}$ referring the dry weight measured after drying the swollen sample during two days at $50{ }^{\circ} \mathrm{C}$.

Rheology: The viscoelastic properties of hydrogels were studied from the preparation state, using a stress-controlled rheometer (DHR3 from TA Instruments) equipped with a roughened plate/plate geometry (diameter $20 \mathrm{~mm}$, gap of $2 \mathrm{~mm}$ ) and temperature controller by a Peltier system. All the experiments were performed in the linear viscoelastic regime at $0.1 \%$ strain amplitude. All the hydrogel samples were immersed in paraffin oil during the experiments to avoid drying. The GN2D3 samples were heated at $60^{\circ} \mathrm{C}$ for $30 \mathrm{~min}$ to avoid the volume change during the temperature-dependent experiment. Frequency sweeps were performed with the angular frequency from 1 to $100 \mathrm{rad} / \mathrm{s}$. Temperature sweeps were performed at $1 \mathrm{rad} / \mathrm{s}$ and a heating rate of $0.25{ }^{\circ} \mathrm{C} / \mathrm{min}$ from $20^{\circ} \mathrm{C}$ to $60{ }^{\circ} \mathrm{C}$.

Tensile tests for large strain behavior: Tensile tests were performed on a standard tensile Instron machine model 5565, equipped with a $10 \mathrm{~N}$ load cell (with a relative uncertainty of $0.16 \%$ in the range from 0 to $0.1 \mathrm{~N}$ ) and an environmental chamber with temperature controller. The hydrogel samples were then cut with a punch and their dimensions were $30 \mathrm{~mm} \times 5 \mathrm{~mm}$ x $2 \mathrm{~mm}$. The gauge length was recorded before each tensile experiment. For high temperature experiment, hydrogel samples were immersed in paraffin oil bath and stabilized at designated temperature for $20 \mathrm{~min}$. The GN2D3 samples were heated at $60{ }^{\circ} \mathrm{C}$ for $20 \mathrm{~min}$ and their final dimensions were collected as $26 \mathrm{~mm}$ x $4.5 \mathrm{~mm}$ x $1.6 \mathrm{~mm}$. All the tests were carried out at the same strain rate of $0.06 \mathrm{~s}^{-1}$.

Nominal stress was defined as $\sigma=\mathrm{F} / \mathrm{S}_{0}$, with $\mathrm{F}$ being the recorded force and $\mathrm{S}_{0}$ being the initial cross section. Reduced stress, $\sigma_{R}$ was calculated by the following equation of $\sigma_{R}=\sigma /\left(\lambda-\lambda^{-2}\right)$, with $\sigma$ being the nominal stress and $\lambda$ being the elongation calculated by $\lambda=L / L_{0}=1+\sigma$.

Fracture tests of notched samples. Fracture tests were performed using the single edge notch geometry on a standard tensile Instron machine model 5565 equipped with a $10 \mathrm{~N}$ load cell. A notch of approximately $1 \mathrm{~mm}$ length was made on the edge of hydrogel strips. The fracture energy, $G_{c}$ has been calculated by the following equation ${ }^{2}: G_{c}=(6 \cdot W \cdot c) / \sqrt{\lambda_{c}}$ with $c$ being the initial notch length, $\lambda_{\mathrm{c}}$ being the fracture strain and $\mathrm{W}$ being the strain energy density calculated by integration of the stress-strain curve.

\section{Preparation of hydrogels with stimuli-responsive gelation.}


Aqueous solution of chitosan $(0.02 \mathrm{~g} / \mathrm{mL})$ was prepared by dissolving TVPA and chitosan in deionized water and the $\mathrm{pH}$ was tuned to 5.6 by the addition of acetic acid. The sodium hydroxide solution $(\mathrm{NaOH}, 0.01 \mathrm{M})$ was poured slowly into the chitosan solution and then the gelation of chitosan was induced by the diffusion of $\mathrm{NaOH} .{ }^{3}$

Aqueous solutions of PEG $(0.013 \mathrm{~g} / \mathrm{mL})$ and $\alpha-\mathrm{CD}(0.145 \mathrm{~g} / \mathrm{mL})$ was prepared by dissolving PEG and $\alpha$-CD with TVPA in deionized water at $50{ }^{\circ} \mathrm{C}$, respectively. The equal volume of PEG and $\alpha$-CD solutions was mixed at $50{ }^{\circ} \mathrm{C}$ and the mixture was gelled after standing at $20^{\circ} \mathrm{C}$ for 24 h. ${ }^{4}$

Aqueous solution of PEG-PPG-PEG (18 wt\%) was prepared by dissolving TVPA and PEGPPG-PEG in deionized water at $90{ }^{\circ} \mathrm{C}$ and cooled down at room temperature. The fluorescent photos of PEG-PPG-PEG solutions at $5{ }^{\circ} \mathrm{C}$ and $20^{\circ} \mathrm{C}$ was taken under UV light. Then PEGPPG-PEG solution was gelled along the glass inner wall by stirring the glass vials slowly in the temperature-controlled oven. ${ }^{5}$
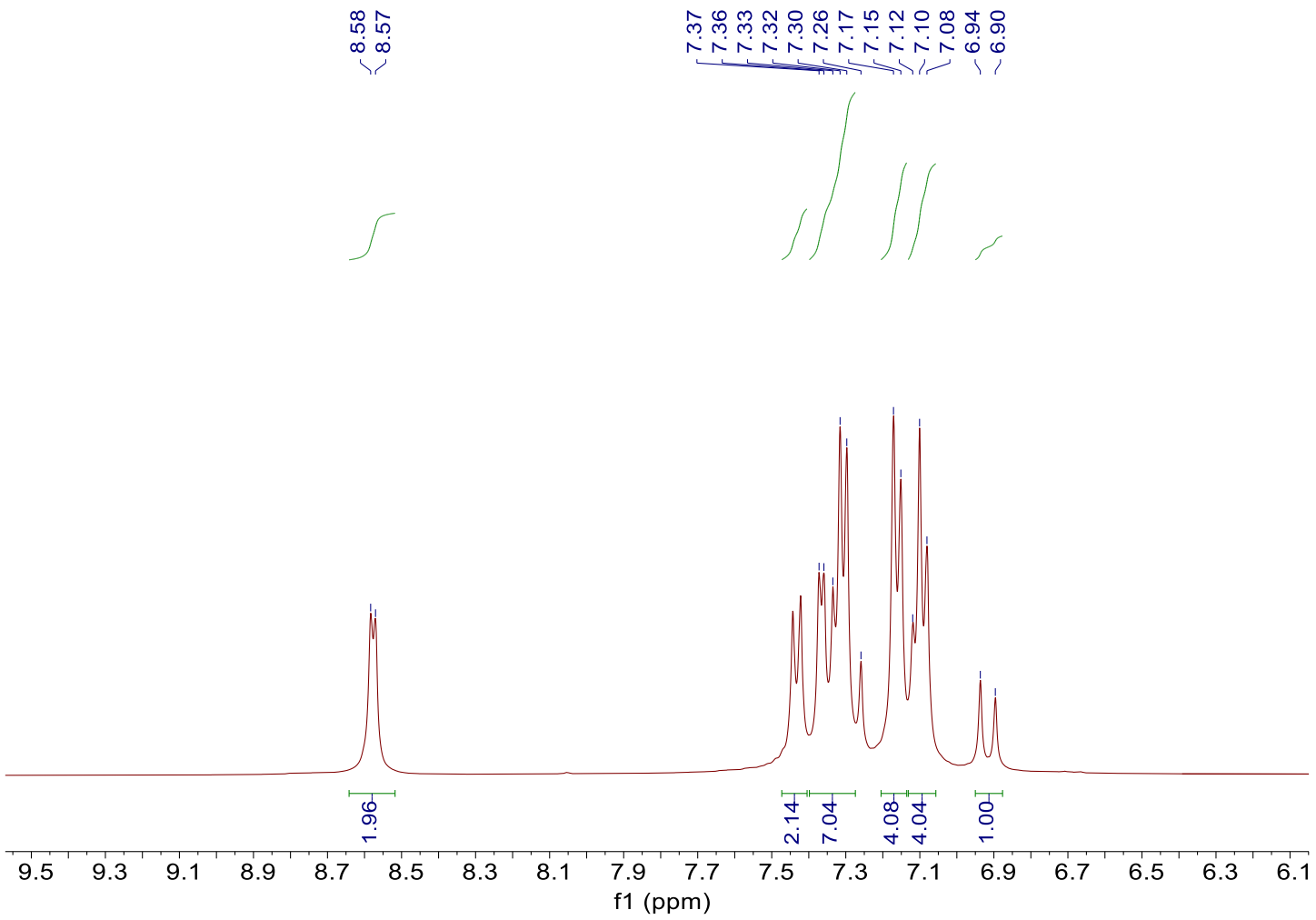

Figure S3. ${ }^{1} \mathrm{H}$ NMR spectrum of $\mathbf{M 1}$ in $\mathrm{CDCl}_{3}$. 


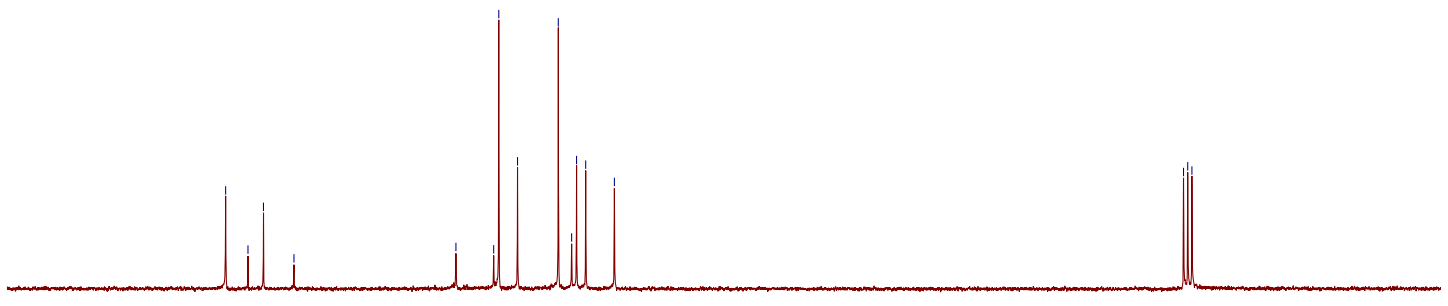

$\begin{array}{llllllllllllllllllllll}165 & 160 & 155 & 150 & 145 & 140 & 135 & 130 & 125 & 120 & 115 & 110 & 105 & 100 & 95 & 90 & 85 & 80 & 75 & 70 & 65 & 60\end{array}$ f1 (ppm)

Figure S4. ${ }^{13} \mathrm{C}$ NMR spectrum of $\mathbf{M 1}$ in $\mathrm{CDCl}_{3}$.

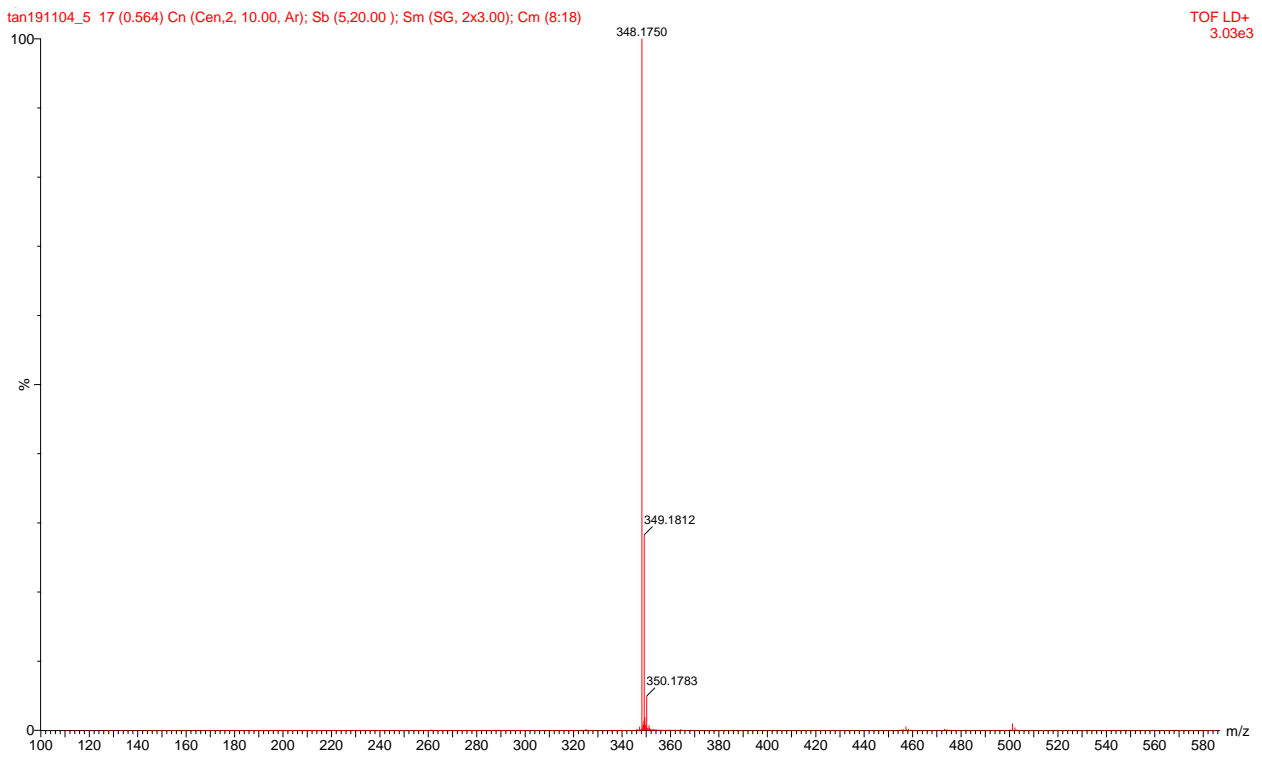

Figure S5. Mass spectrum of M1. 

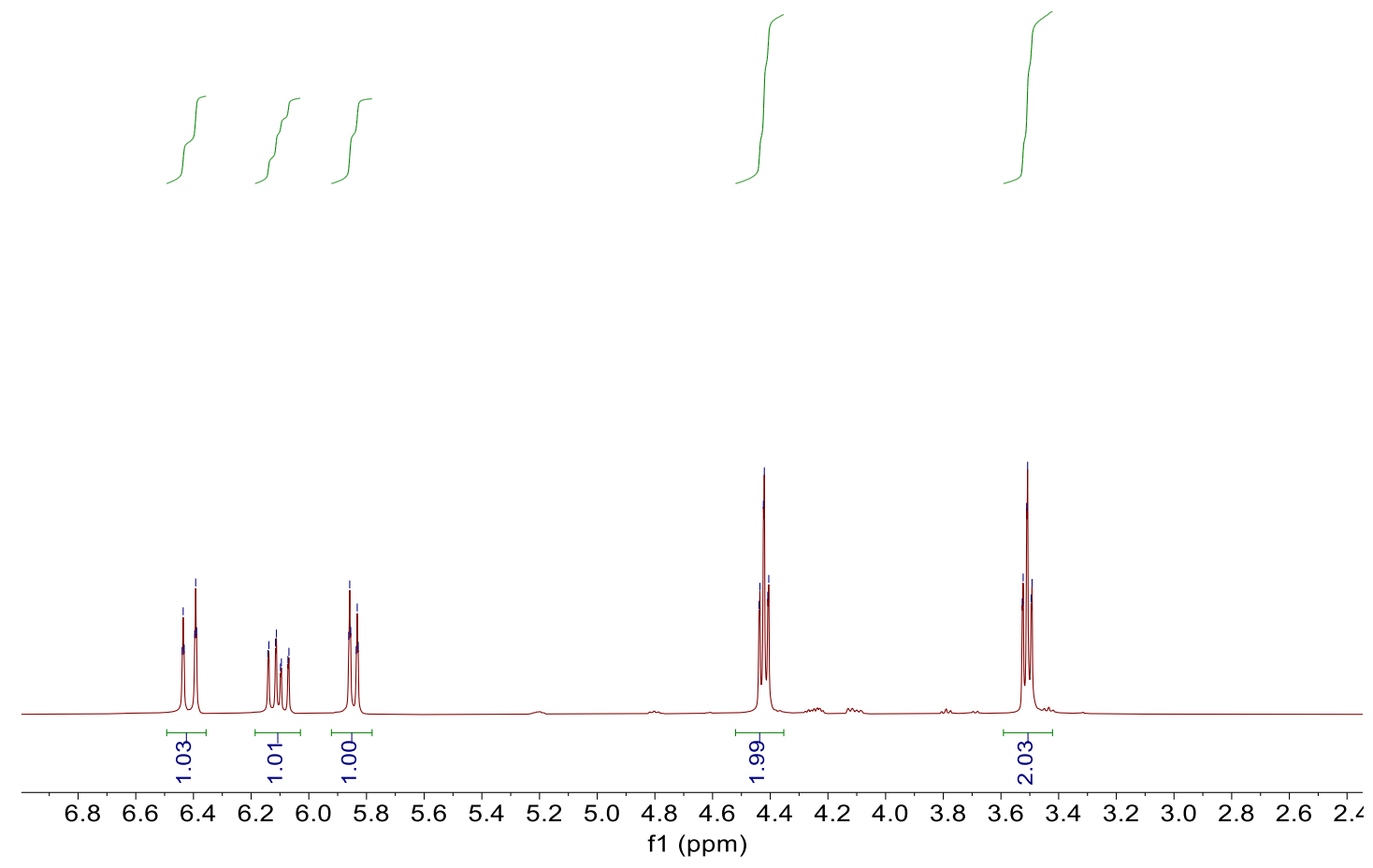

Figure S6. ${ }^{1} \mathrm{H}$ NMR spectrum of $\mathbf{M} 2$ in $\mathrm{CDCl}_{3}$.

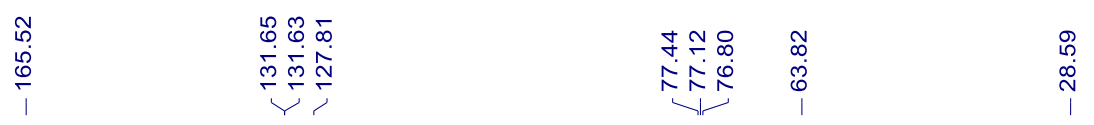

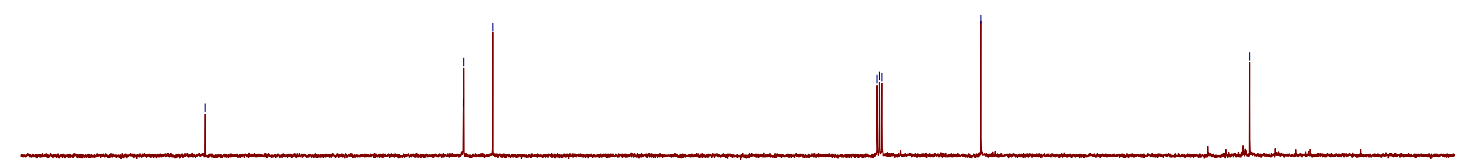

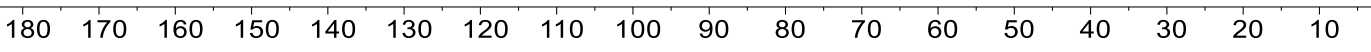
f1 (ppm)

Figure S7. ${ }^{13} \mathrm{C}$ NMR spectrum of $\mathbf{M} 2$ in $\mathrm{CDCl}_{3}$. 


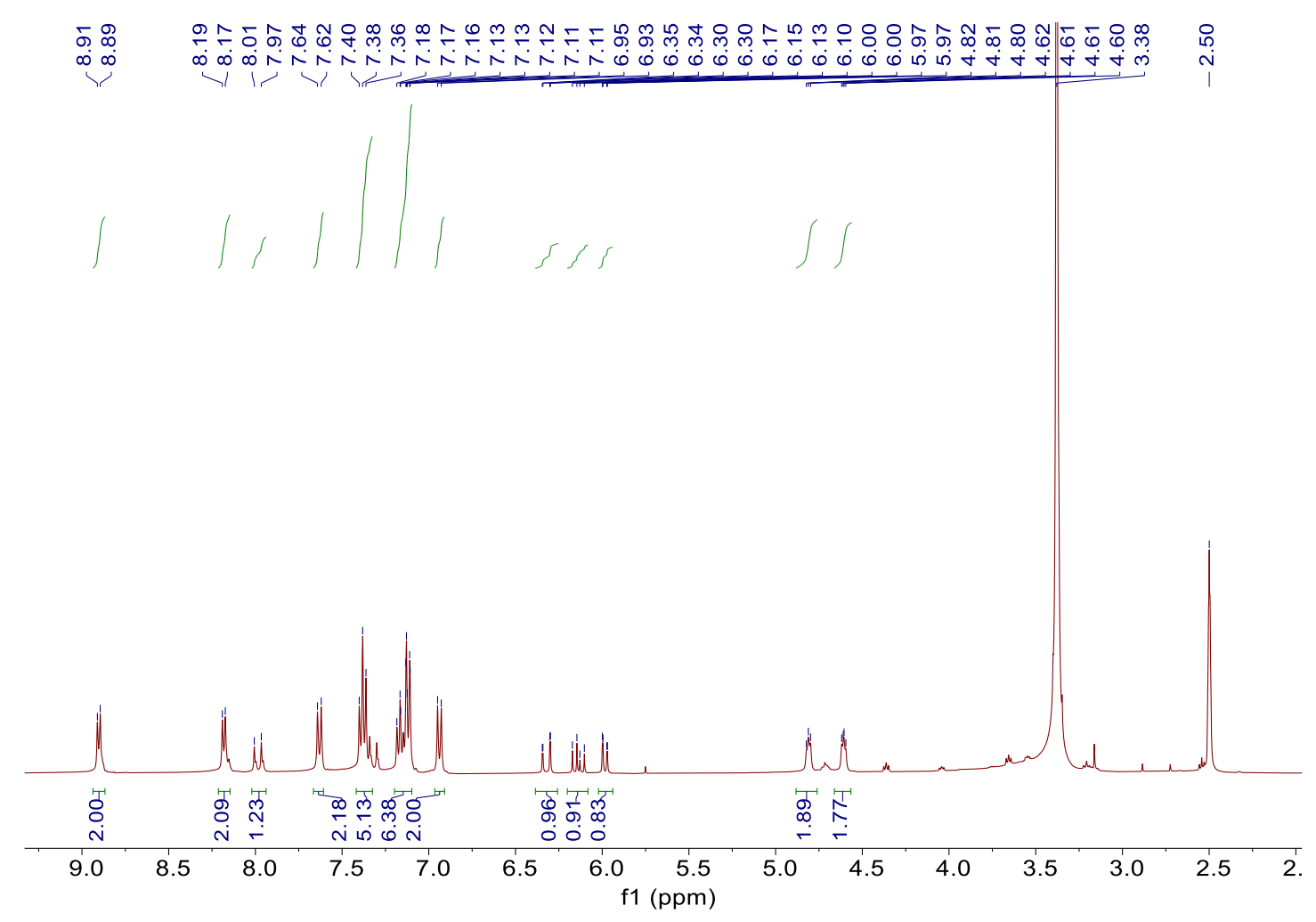

Figure 8. ${ }^{1} \mathrm{H}$ NMR spectrum of TVPA in DMSO- $d 6$.

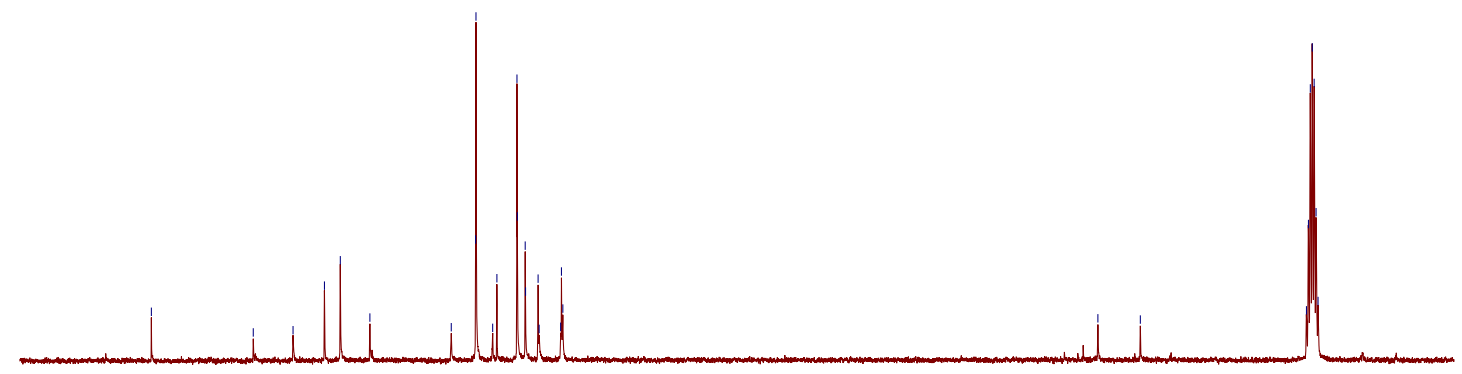

$\begin{array}{llllllllllllllll}175 & 165 & 155 & 145 & 135 & 125 & 115 & 105 & 95 & 85 & 75 & 65 & 55 & 45 & 35 & 2\end{array}$

Figure S9. ${ }^{13} \mathrm{C}$ NMR spectrum of TVPA in DMSO- $d_{6}$. 


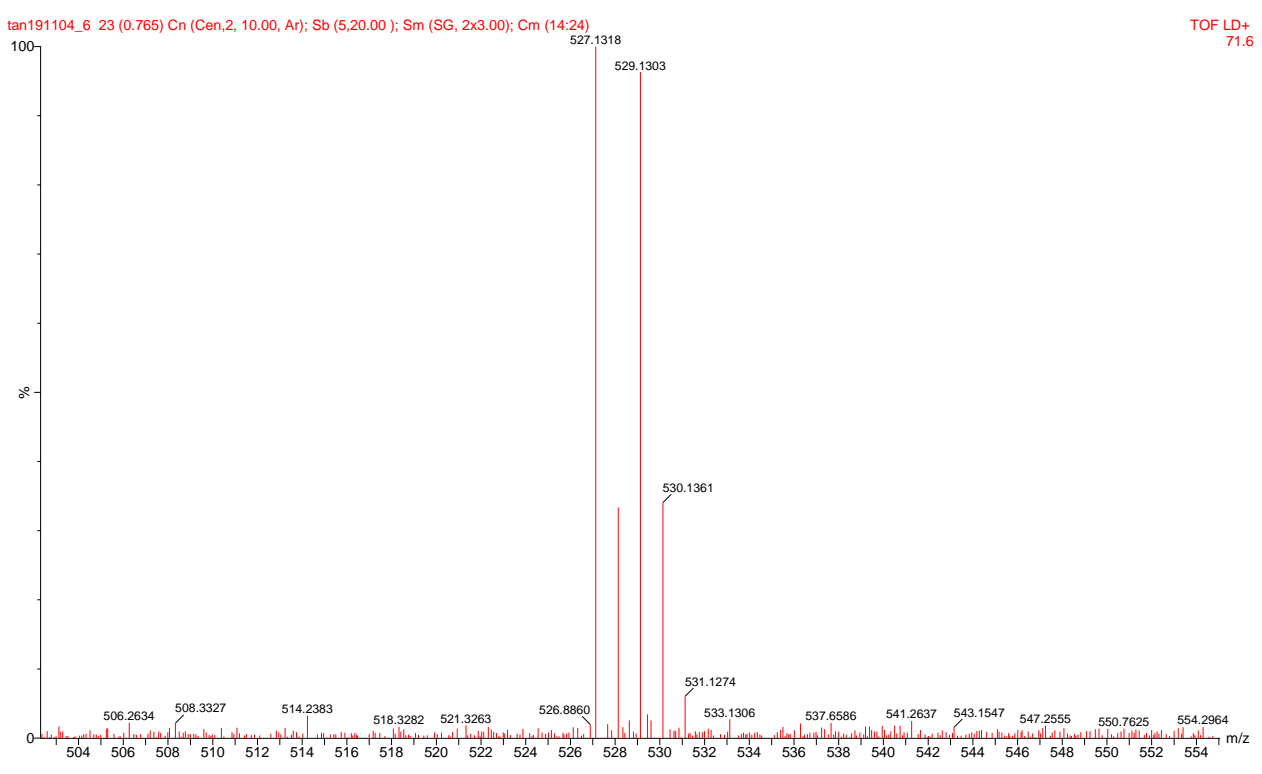

Figure S10. Mass spectrum of TVPA.
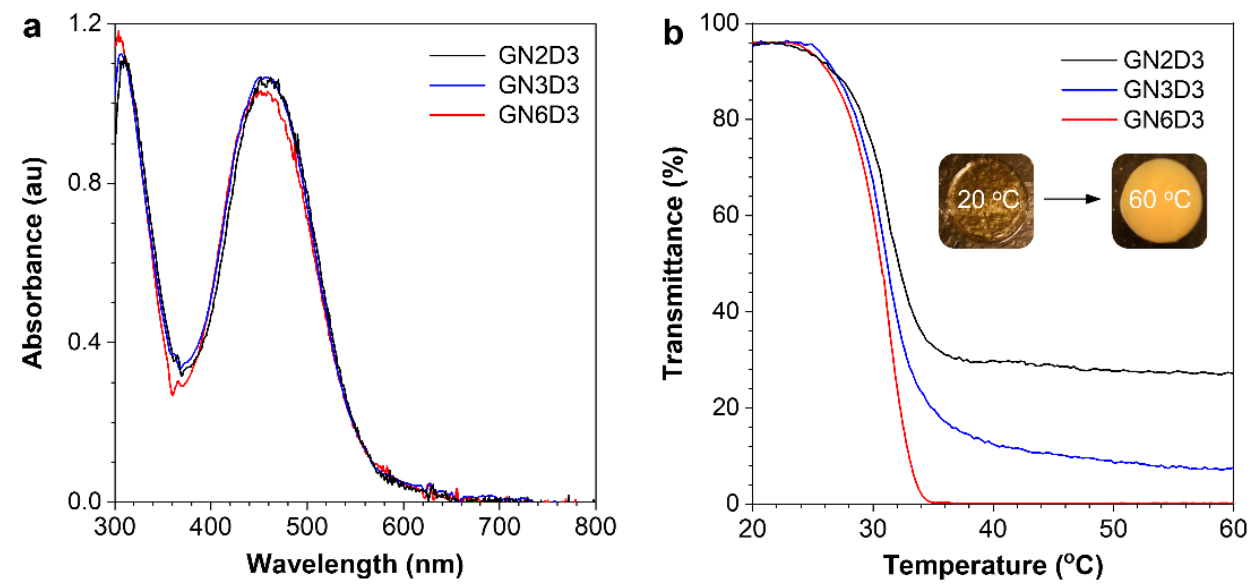

Figure S11. a, UV-vis spectra of GN2D3, GN3D3 and GN6D3 in $20{ }^{\circ} \mathrm{C}$. b, Temperaturedependent transmittance of GN2D3, GN3D3 and GN6D3 from $20{ }^{\circ} \mathrm{C}$ to $60^{\circ} \mathrm{C}$ with a heating rate of $0.3{ }^{\circ} \mathrm{C} / \mathrm{min}$ determined by absorption intensity at $600 \mathrm{~nm}$. 

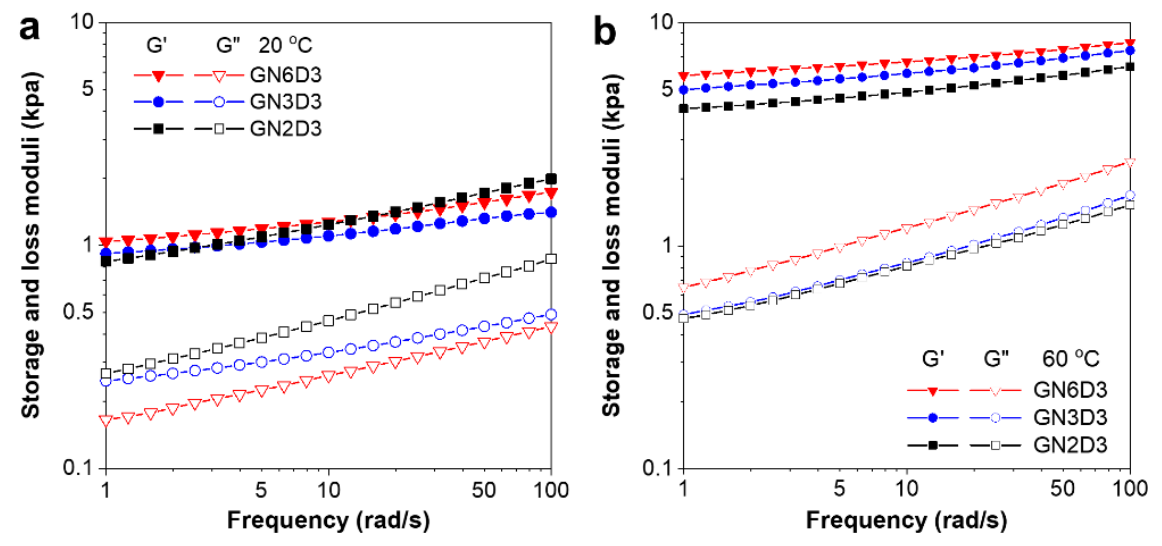

Figure S12. Frequency dependent storage (solid symbols, G') and loss (open symbols, G”) moduli of GN2D3, GN3D3 and GN6D3 at $20{ }^{\circ} \mathrm{C}(\mathbf{a})$ and at $60{ }^{\circ} \mathrm{C}(\mathbf{b})$.
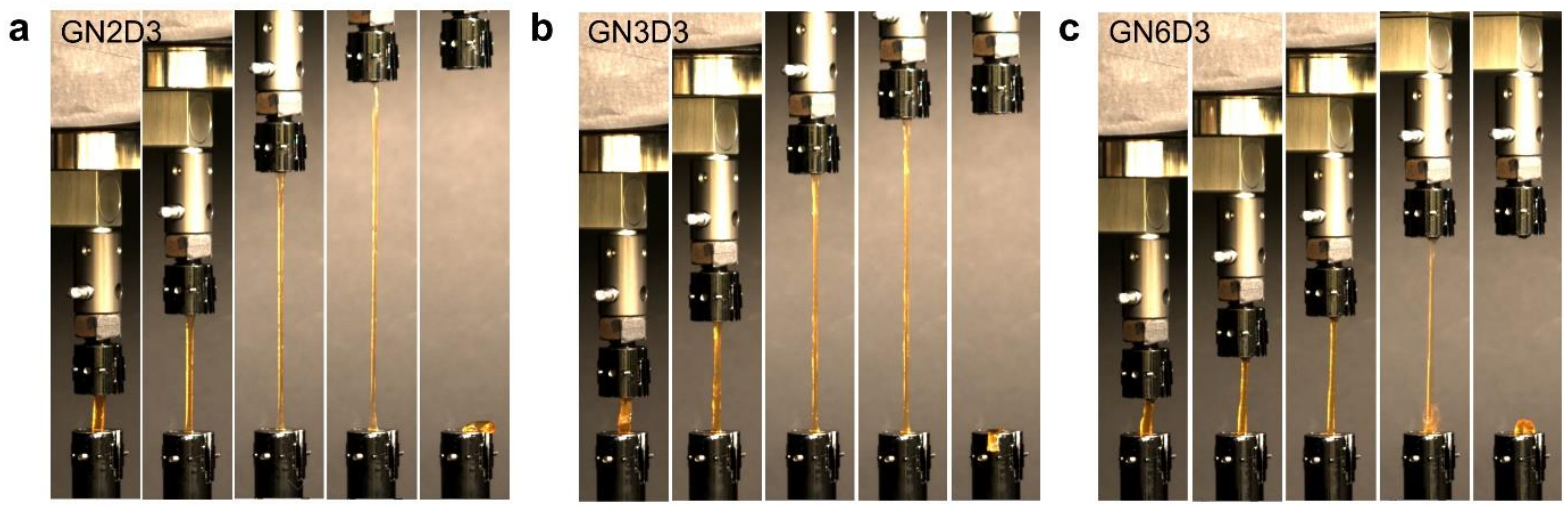

Figure S13. Tensile tests of GN2D3, GN3D3 and GN6D3 at $20^{\circ} \mathrm{C}$.

a

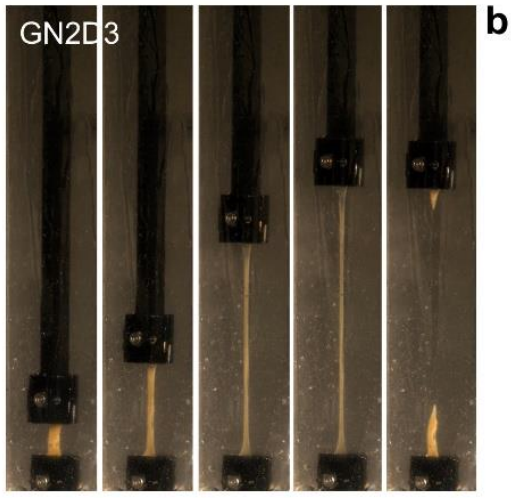

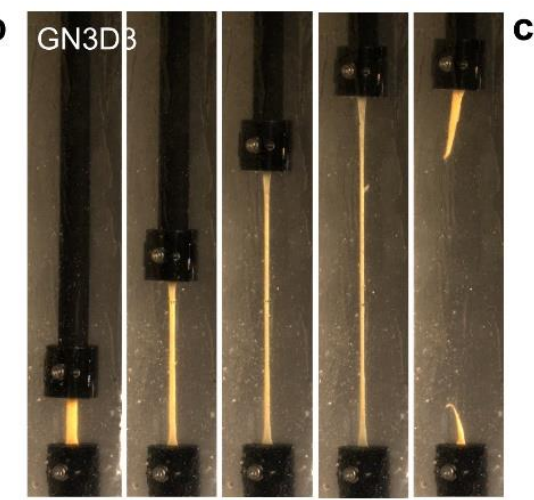
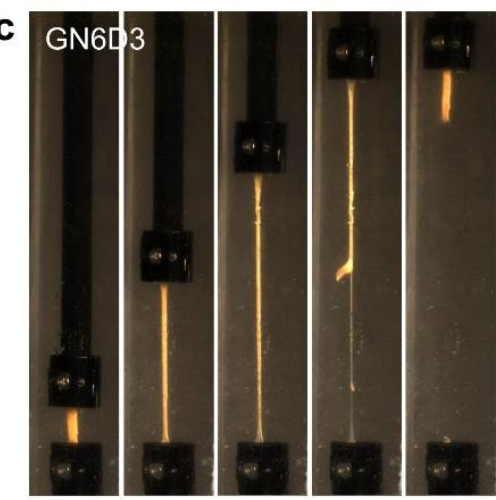

Figure S14. Tensile tests of GN2D3, GN3D3 and GN6D3 in paraffin oil baths at $60{ }^{\circ} \mathrm{C}$. 


\section{References}

1. T. R. Barlow, J. C. Brendel and S. Perrier. Poly(bromoethyl acrylate): A Reactive Precursor for the Synthesis of Functional RAFT Materials. Macromolecules 2016, 49, 6203-6212.

2. H. W. Greensmith, Rupture of rubber. X. The change in stored energy on making a small cut in a test piece held in simple extension. J. Appl. Polym. Sci. 1963, 7, 993-1002.

3. Y. Yang, X. Wang, F. Yang, H. Shen, and D. Wu. A universal soaking strategy to convert composite hydrogels into extremely tough and rapidly recoverable double-network hydrogels. Adv. Mater. 2016, 28, 7178-7184.

4. J. Li, A. Harada and M. Kamachi. Sol-gel transition during inclusion complex formation between $\alpha$-cyclodextrin and high molecular weight poly(ethylene glycol)s in aqueous solution. Polym. J. 1994, 26, 1019-1026.

5. S. Y. Lee, Y. Lee, J. E. Kim, T. G. Park and C. H. Ahn. A novel pH-sensitive PEG-PPGPEG copolymer displaying a closed-loop sol-gel-sol transition. J. Mater. Chem. 2009, 19, 8198-8201. 
